\title{
Synthesis and Antitumor Evaluation of Novel Cyclic Arylsulfonylureas: ADME-T and Pharmacophore Prediction
}

\author{
Ibrahim M. El-Deeb ${ }^{\mathrm{a}}$, Said M. Bayoumia ${ }^{\mathrm{a}}$, Magda A. El-Sherbeny ${ }^{\mathrm{a}}$, \\ Alaa A.-M. Abdel-Aziz ${ }^{\mathrm{a}, \mathrm{b}, *}$ \\ Department of Medicinal Chemistry ${ }^{a}$, Faculty of Pharmacy, University of Mansoura, Mansoura 35516, Egypt \\ Department of Pharmaceutical Chemistry ${ }^{b}$, College of Pharmacy, King Saud University, Riyadh 11451, Saudi Arabia
}

Elsevier Use Only: Received date here; revised date here; accepted date here

\begin{abstract}
Novel derivatives of 5-(substituted)benzylidene-3-(4-substituted)phenylsulfonylimidazolidine-2,4-diones (3ar), 1-(4-substituted)phenylsulfonyl-3-(4-substituted)phenylpyrimidine-2,4,6-(1H,3H,5H)-triones (6a-l), and 3-(4-substituted) phenyl-1-(4-substituted)phenylsulfonylquinazoline-2,4(1H,3H)-diones (8a-l) have been synthesized and tested for their antitumor activity against 60 tumor cell lines taken from 9 different organs. The tested compounds have showed good inhibitory effect at the ovarian cancer (IGROV1) cell line. A significant inhibition for (RXF393) renal cancer cells was observed with series $\mathbf{3}$ compounds, while in the other two series $\mathbf{6}$ and $\mathbf{8}$, there was a significant inhibition of ovarian cancer cells (OVCAR-8) and melanoma cells (SK-MEL-2). Interestingly; beside the strong inhibition of compound 3q to IGROV1 and RXF393 cells, a great inhibition (199.62 \%) for (M14) Melanoma cells was observed at the tested concentration (10 $\mu$ M). ADME-T and pharmacophore prediction methodology were used to study the antitumor activity of the most active compounds and to identify the structural features required for antitumor activity. (C) 2011 Elsevier Science. All rights reserved.
\end{abstract}

Keywords: Cancer; Molecular Modeling; Imidazolidine; Pyrimidine; Quinazoline; Diarylsulfonylurea; Pharmacophore.

\section{Introduction}

Cancer is continuing to be a major health problem in developing as well as developed countries $[1,2]$. Surpassing heart diseases, it is taking the position number one killer due to various worldwide factors.

\footnotetext{
* Corresponding author. Tel.: 00966-56-2947305; fax: 00966-1-4676220; e-mail: alaa_moenes@yahoo.com
} 
Although major advances have been made in the chemotherapeutic management of some patients, the continued commitment to the laborious task of discovering new anticancer agents remains critically important. Among the wide range of compounds tested as potential anticancer agents, derivatives comprising the sulfonamide, diarylsulfonylurea and thiourea functionalities have attracted reasonable attention [3-8], especially after the discovery of sulofenur [9] (LY186641) A and its structure analogs [8] B and (LY295501) C (Figure 1). Sulofenur is an antineoplastic sulfonylurea that has been clinically evaluated in lung, breast, colon, ovarian, pancreatic, and gastric cancer [9]. It is generally assumed that the strong cytotoxicity and, as a consequence, the antitumor properties of the diarylsulfonylurea is due to the uncoupling of mitochondria [4,5], however, other mechanisms, such as inhibition of the mitochondrial isozyme V of carbonic anhydrase (CAs) have also been hypothesized, since hydrolysis of the cytotoxic agent leading to the formation of unsubstituted sulfonamides as the principal products has been reported both in vivo and in vitro [10]. It is well known that aromatic/heterocyclic sulfonamides (formed after such a hydrolytic process) act as very potent inhibitors of CAs [11,12], and that these enzymes are involved in a multitude of crucial physiologic processes [13]. However, clinical trials of sulofenur have yielded unsatisfactory results because of its high protein binding and limited dosing caused by the appearance of anemia, and methemoglobinemia, a side effect that is likely caused by its aniline-related metabolites [14]. In contrast; LY295501 (C) is principally metabolized by hydroxylation with negligible formation of aniline metabolites at relevant doses in experimental animals and demonstrated impressive activity against a broad spectrum of human tumor xenografts [15].

Trying to overcome the serious side effects of Sulofenur, several imidazolidinone derivatives containing diarylsulfonylurea pharmacophore, have been synthesized and screened for antitumor activity against various human solid tumors. Among these compounds are; 1-(4-clorophenylsulfonyl)-4phenylimidazolidin-2-one (D) and DW2143 (E) which have shown higher cytotoxic activity than that of Sulofenur, in addition, there was no production of methemoglobinemia or hypoglycemia upon 
administration of these agents, which indicates a different metabolic fate from that of Sulofenur (Figure 1) [16-26]. On the other hand, over the past few years, much interest has been given to the chemotherapeutic activity of hydantoins [27-31]. Quinazolinediones [32,33] and pyrimidinetriones $[34,35]$ as potential anticancer agents.

In view of the previous rationale and in continuation of an ongoing program aiming at finding new structure leads with potential chemotherapeutic activities, new series of 5-(substituted) benzylidene-3(4-substituted)phenylsulfonylimidazolidine-2,4-diones (3a-r), 1-(4-substituted) phenylsulfonyl-3-(4substituted)phenylpyrimidine-2,4,6-(1H,3H,5H)-triones $\quad \mathbf{( 6 a - 1 ) , \quad \text { and } \quad 3 - ( 4 - s u b s t i t u t e d ) p h e n y l - 1 - ( 4 -}$ substituted)phenylsulfonylquinazoline-2,4(1H,3H)-diones (8a-l) have been synthesized and screened for cytotoxic activity (Figure 1). These series comprise the derived $N^{1}, N^{3}$-disubstituted sulfonylurea pharmacophores that are structurally related to sulofenur $\mathbf{A}$ and its cyclic form $\mathbf{D}$ (Figure 1). The thrust of efforts in the derivatization of such type of compounds focused mainly on the aryl moiety of the sulfonamide portion of the diarylsulfonylureas. In the present study, such arylsulfonylurea moiety was incorporated basically as a part of the cyclic structures of hydantoin because of the reported potential anticancer activity of this ring system hoping to induce some biological synergism. In addition, ring replacement of the hydantoin with pyrimidinetrione and quinazolinedione functionality was considered as an interesting structure variation in order to study the influence of such modification on the anticipated antitumor activity. The substitution pattern at the aryl part of the diarylsulfonylurea pharmacophores was selected so as to confer different electronic environment that would affect the lipophilicity, and hence the activity of the target molecules. The objective of forming these hybrids is an attempt to reach an active antitumor agent with potentiated activity and selectivity toward cancerous cells. Moreover ADME-T and pharmacophore prediction methodology were used to identify the structural features required for the antitumor properties of these new series. 


\section{Results and discussion}

\subsection{Chemistry}

\subsubsection{Synthesis of compounds 3a-r (Scheme 1, Table 1).}

Scheme 1 outlines the synthetic pathway used to obtain compounds (3a-r). The starting material 5(substituted)benzylideneimidazolidine-2,4-diones (2a-f) were prepared by allowing hydantoin $\mathbf{1}$ to condense with the corresponding aromatic aldehydes in glacial acetic acid and in the presence of fused sodium acetate as a condensing agent [36]. Upon mixing of $\mathbf{2}$ with the appropriate arylsulfonyl chloride and $\quad \mathrm{K}_{2} \mathrm{CO}_{3} \quad$ in $\quad$ acetone:water (1:1); 5-(substituted)benzylidene-3-(4-substituted) phenylsulfonylimidazolidine-2,4-diones (3a-r) were obtained in relatively good yield. Although geometrical isomerism ( $E / Z$ isomers) was possible because of the restricted rotation about the exocyclic $\mathrm{C}=\mathrm{C}$ bond of the arylidene hydantoins, all the derivatives prepared in this study were obtained exclusively in the Z-form as confirmed by the analytical data. In all the IR spectra of the prepared arylidenehydantoins (2a-f), and their subsequent arylsulfonyl derivatives (3a-r), the stretching of the $\mathrm{C}=\mathrm{C}$ bond appeared in a higher frequency region $\left(1660-1675 \mathrm{~cm}^{-1}\right)$ compared to those expected for the E-form (1630-1640 $\mathrm{cm}^{-1}$ ) [37]. Furthermore; the $\mathrm{H}^{1}$-NMR spectra of the prepared arylidene hydantoins have showed that the most diagnostic olefinic proton, $\mathrm{H} 6(-\mathrm{CH}=)$ was deshielded more $(\delta=6.4-7.0 \mathrm{ppm})$ as expected in the $Z$-form, relative to the slightly shielded proton of the $E$-form ( $\delta=6.2-6.3 \mathrm{ppm})$. This deshielding of the olefinic proton is caused by the anisotropic effect exerted by the nearby C4 carbonyl group in the Z-isomer [37].

\subsubsection{Synthesis of compounds 6a-l (Scheme 2, Table 2).}

1-(4-substituted)phenylureas (4a-d); which were prepared according to Hofmann [38,39] procedure by reacting the aromatic amine with potassium cyanate in water and in the presence of excess acetic acid; were treated with diethyl malonate in the presence of sodium ethoxide/absolute ethanol to afford 1-(4substituted)phenylpyrimidine-2,4,6-(1H,3H,5H)-triones (5a-d) [40]. As previously mentioned under the 
preparation of compounds 3a-r; compounds 6a-l were prepared by addition of the appropriate arylsulfonyl chloride to a stirred solution of 5a-d and $\mathrm{K}_{2} \mathrm{CO}_{3}$ in acetone: water (1:1).

\subsubsection{Synthesis of compounds 8a-l (Scheme 3, Table 3).}

3-(4-substituted)phenylquinazoline-2,4(1H,3H)-diones (7a-d) were prepared by fusing anthranilic acid with 1-(4-substituted)phenylureas (4a-d) [41]. The synthesis of 3-(4-substituted)phenyl-1-(4substituted)phenylsulfonylquinazoline-2,4(1H,3H)-diones (8a-l) was performed via the reaction of 3-(4substituted)phenylquinazoline-2,4(1H,3H)-diones (7a-d) with sodium tert-butoxide in tert-butanol to induce salt formation, then the separated salt was refluxed with an equivalent amount of the appropriate arylsulfonyl chloride in tert-butanol to yield the target products $\mathbf{8 a}-\mathbf{l}$.

\subsection{Biological activity}

\subsubsection{In vitro antitumor evaluation}

The Thirteen compounds indicated in (Figures 2 and 3; Tables 4 and 5) were selected by National Cancer Institute, Bethesda, Maryland, USA on the basis of degree of the structure variation and computer modeling techniques for evaluation of their antineoplastic activity. The selected compounds were subjected to in vitro anticancer assay against tumor cells in a full panel of 60-cell lines taken from 9 different organs (lung, colon, breast, ovary, blood, kidney, skin, prostate and brain). The compounds were tested at a single dose concentration of $10 \mu \mathrm{M}$, and the percentages of growth inhibitions over the sixty tested cell lines were determined. The percentages of growth inhibitions over the most sensitive 5cell lines are shown in figure 2 and table 4.

\subsubsection{Structural activity relationship}

By investigating the variation in selectivity of the tested compounds over the full panel of cell lines, it was revealed that nearly all of the compounds belonging to the three series (compounds 3, 6 \& 8) show significant inhibition for the ovarian cancer cell line (IGROV1). The percentages of inhibition for (IGROV1) ovarian cancer reached $100 \%$ in a number of the tested derivatives (Figure 2, Table 4). 
However; a distinguish in selectivity was observed between the first series (compounds 3), and the second and third series (compounds $\mathbf{6}$ and $\mathbf{8}$ ). In series $\mathbf{3}$, a significant inhibition for (RXF393) renal cancer cells (76.2 - $96.5 \%$ ) was observed, while in both of the other two series $\mathbf{6}$ and $\mathbf{8}$, there was a significant inhibition for another type of ovarian cancer cells (OVCAR-8; 33.2 - 58.9 \%) and melanoma cells (SK-MEL-2; 61.0 - $79.3 \%$ ). The agreement between the three series in the inhibition of (IGROV1) ovarian cancer cells could be correlated to a similar inhibitory mechanism related to the common structural feature in the three series (the diarylsulfonyl urea structure), while the variation in selectivity over RXF393, OVCAR-8 and SK-MEL-2 cell lines is probably caused by the differences in the hydrocarbon skeleton holding the cyclic urea structure in the three series. These variations could be also correlated to the small difference in the distance between the cores of the two aryl moieties of the diarylsulfonylurea structure, where this distance is almost the same in compounds $\mathbf{6 a - l}$ and $\mathbf{8 a}-\mathbf{l}$, while a little bit longer in compounds 3a-r. This variation in distance can account for the similar inhibitory profiles for series $\mathbf{6}$ and $\mathbf{8}$, and its variation in the compounds belonging to series $\mathbf{3}$. An odd result was observed with compound 3q (Figure 3, Table 5), where beside the strong inhibition of IGROV1 cells (97.89 \%) and RXF393 cells (95.74 \%), a great inhibition (199.62 \%) for M14 Melanoma cells was observed at the tested concentration $(10 \mu \mathrm{M})$. This great inhibition at the mentioned concentration indicates a great potency for the compound with a strong lethal effect over (M14) melanoma cells (99.6\% lethality), and suggests an $\mathrm{IC}_{50}$ value in the nanomolar scale.

\subsection{Molecular modeling Results}

Modeling studies are required in order to construct molecular models that incorporate all experimental evidence reported. These models are necessary to obtain a consistent and more precise picture of the biological active molecules at the atomic level and furthermore, provide new insights that can be used to design novel therapeutic agents.

\subsubsection{Conformational analysis}


In an attempt to gain a better insight on the molecular structures of the active compounds $\mathbf{3 p}$, $\mathbf{3 q}, \mathbf{6 k}$ and $\mathbf{8 b}$ compared with the least active species $\mathbf{3 b}, \mathbf{6 b}$ and $\mathbf{8 j}$ as representative examples; conformational analysis of the target compounds has been performed using the MMFF94 force-field [42,43] (calculations in vacuo, bond dipole option for electrostatics, Polak-Ribiere algorithm, RMS gradient of $0.01 \mathrm{kcal} / \AA \AA \mathrm{mol}$ ) implemented in MOE 2009.10 [44]. The most stable conformer was fully optimized by AM1 [45] semi-empirical molecular orbital calculation (Figure 4). Calculations at the AM1 level were considered in order to determine relative energies of the $E$ - and Z-isomers of 5-arylidenehydantoin derivatives (3). It was found that the $Z$-isomer is approximately more stable by more than $3 \sim 4 \mathrm{kcal} / \mathrm{mol}$ and thus no double bond isomerisation is anticipated (Figure 4) [46]. Moreover the bond distances between $\mathrm{N}$ and $\mathrm{SO}_{2}$ was found to be $1.67 \AA$, and a twisting of c.a. $34^{\circ}$ between the arylidene moiety and hydantoin plane was also found [46]. As clear from the calculations; series 3 compounds exhibit structural similarity as indicated by their molecular parameters and are slightly different from series 6 and 8 compounds. The results showed that the lowest energy minimized structures of compounds belonging to the three series have exhibited the same arrangement of the arylsulfonyl groups (Figures 4 and 5) around the cyclic urea moiety in which the arylsulfonyl group was out of the plane of cyclic urea group ( $\angle \mathrm{N}-\mathrm{CO}-\mathrm{N}-\mathrm{SO} 2$ torsional angle was $177^{\circ}, 155^{\circ}, 133^{\circ}$ in case of series $\mathbf{3}, \mathbf{6}$ and $\mathbf{8}$ compounds, respectively).

\subsubsection{ADME-T prediction}

\subsubsection{Lipinski's rule of five and the effect of lipophilic and steric parameters}

As a part of our study; the compliance of compounds to the Lipinski's rule of five was evaluated [47]. In addition, the polar surface area (PSA) of the compounds was also calculated (Table 6), since it is another key property that has been linked to drug bioavailability, where passively absorbed compounds with a PSA $>140 \AA 2$ are thought to have low oral bioavailability [48]. The results disclosed in table 6 show that all of the synthesized compounds comply with these rules. Hence; theoretically, all of these 
compounds should present good passive oral absorption and differences in their bioactivity cannot be attributed to this property.

The introduction of cyclic ring fragments incorporating the arylsulfonylurea core and the variation of ring size and the substituents on these fragments have allowed us to evaluate the influence of lipophilicity and steric parameters at the pharmacophoric part of the molecules. Table 6 gathers cytotoxic activity against ovarian human cell line (IGROV1) as a representative example as well as values of ClogP (lipophilic factors), molar refractometry and polar surface area (steric factors) for each compound, determined by using MOE program. Within the series of compounds 3a-d we have observed sharp increase in the antitumor activity with the increase in molar refractometry from $88.7 \mathrm{~cm}^{3} / \mathrm{mol}$ (3b and $\mathbf{3 g}$ ) to $99.3 \sim 103.9 \mathrm{~cm}^{3} / \mathrm{mol}$ (3p and 3q). This effect of molar refractometry was observed within the other two series too, such as in compounds $\mathbf{6 b}, \mathbf{6 k}, \mathbf{8 a}$ and $\mathbf{8 b}$ with molar refractometris of $86.4 \mathrm{~cm}^{3} / \mathrm{mol}^{\text {, }}$ $93.7 \mathrm{~cm}^{3} / \mathrm{mol}, 98.1 \mathrm{~cm}^{3} / \mathrm{mol}$ and $102.7 \mathrm{~cm}^{3} / \mathrm{mol}$ respectively. Although lipophilicity does not exert a significant effect on activity in compounds $\mathbf{3}$ and $\mathbf{6}$, an increase in potency was observed in compounds 8a, 8b \& 8k with $\log \mathrm{P}$ values higher than 2.7. Regarding steric parameters; from the data gathered in table 6 there is a clear influence of refractometry on antitumor activity compared with lipophilicity and polar surface area for either series of all compounds. The optimal refractometry for the most active compounds was found to lie in the range of $91.4 \sim 103.9 \mathrm{~cm}^{3} / \mathrm{mol}$ (Table 6).

\subsubsection{ADME-Tox evaluation $[49,50]$}

To estimate the prospect of series 3, $\mathbf{6}$ and $\mathbf{8}$ compounds as antitumor agents compared with the reported antitumor agents A, D and E; their drug-likeliness were calculated according to absorption, distribution, metabolism, elimination, and toxicity (ADME-T) program, and defined human intestinal absorption (HIA) model [49]. It was predicted that the examined compounds could be transported across the intestinal epithelium, they probably have high affinity to the plasma proteins, and they can cross the blood-brain barrier and are of medium aqueous soluble. The values of HIA, protein binding, BBB 
crossing and solubility prediction for all compounds are presented in Table 7. In general, all compounds presented some advantages and disadvantages when compared to each other. No marked differences in health effects and in rodent toxicity profiles were observed among the compounds. However, the absorption related parameters call for attention, since the promising compounds $\mathbf{3}$ and $\mathbf{6}$ were calculated to be at least as soluble as the reported compounds, and are predicted to have oral bioavailability and absorption significantly higher than that of the reported antitumor agents $\mathbf{A}, \mathbf{D}$ and $\mathbf{E}$. These values are also comparable (marginally inferior or superior) to those obtained from series 8 compounds. The present results suggest an optimal pharmacokinetic profile. Accordingly; it can be deduced from these results that the pharmacokinetic profile of diarylsulfonylureas is affected and modified by the presence of arylsulfonyl moiety connected to heterocyclic ring systems.

\subsubsection{Pharmacophore modelling $[51,52]$}

A molecular modeling experiment was carried out to develop a hypothetical pharmacophore model for the antitumor activity aiming to study the fitting of the designed compounds to this pharmacophore. A ligand-based pharmacophore model was developed using a training set of nine diarylsulfonylureas of diverse chemical structures including compounds A-E (Figure 6), in addition to compounds $\mathbf{3 h}, \mathbf{3 n}, \mathbf{6 b}$ and 8a. The generated hypothetical pharmacophore (Figure 6) showed nine overlapping points with similar chemical properties in the training set; F1: a hydrogen bond acceptor center; F2: an aromatic or hydrophobic center with two parallel places "F3 and F5" for Pi orbital accommodation; F4: an aromatic center with a H-bond donor group; F6: a hydrophobic center; and F7: a second H-bond acceptor center. Fitting of the designed active compounds $\mathbf{3 p}, \mathbf{3 q}, \mathbf{6 k}$ and $\mathbf{8 b}$ to the pharmacophore revealed the presence of the appropriate chemical groups superimposed on the pharmacophoric elements (Figures 6 and 7). The diaryl tail represents the aromatic ring with its Pi orbitals properly oriented to fit to F2, F3 and F5. Also, N-CO-N and C-CO-C of the ring fragment are H-bond acceptors corresponding to F1. The hydrogen bond acceptor sulfonyl group was found to be fit into F6; it is worthy to mention that this 
moiety was perfect in adjusting the distance between the other groups in the molecule. This pharmacophoric assumption was in consistence with the reported results for the antitumor arylsylfonylureas $[26,53,54]$.

\section{Conclusion}

The present work has lead to the development of novel antitumor molecules and some of which have shown promising antitumor activities. As evident from the experimental and calculated data, the structural features (pharmacophores) essential for the antitumor activity of this series are as follows; (1) a cyclic urea that is an essential backbone that carries the recognition feature for biological activity, (2) the presence of arylsulfonyl moiety at the 1-position in case of series $\mathbf{3}$ and the 3-position in case of series $\mathbf{6}$ and $\mathbf{8}$ of cyclic urea core, (3) the two aryl groups of the diarylsulfonylurea skeleton should reside in a certain distance, ranging between $8.7-9.5 \AA$. The new cyclic arylsulfonylureas prepared in this work have good physical properties that qualify them to have good pharmacokinetics and drug bioavailability. They are fully compatible with Lipinski’s rule of five (low molecular weight, favourable ClogP, favourable hydrogen bond-donating and accepting capabilities). They have a simple synthetic access and thus low production costs. Further optimization and pharmacokinetic profiling of these series are currently ongoing.

\section{Experimental}

\subsection{Chemistry}

All melting points $\left({ }^{\circ} \mathrm{C}\right)$ were measured by fisher-johns apparatus and are uncorrected, ${ }^{1} \mathrm{HNMR}$ spectra (TMS as internal standard, chemical shifts in ppm) were measured on a varian EM 360 (200 MHZ) instrument. Thin-layer chromatography (TLC) was conducted on silica gel 60F254 plates (Merck KgaA). Elemental analysis (C,H,N,S) were performed at the microanalytical center, Cairo university, all 
the compounds were within $0.4 \%$ of the theoretical values. Compounds 2a-f [36], 4a-d [38,39], 5a-d [40], and 7a-d [41] have been synthesized according to the reported procedures.

\subsubsection{General procedure for the synthesis of compounds 3a-r.}

A mixture of compounds 2a-f $(0.01 \mathrm{~mol})$ and $\mathrm{K}_{2} \mathrm{CO}_{3}(0.69 \mathrm{~g}, 0.005 \mathrm{~mol})$ was stirred in acetone/water solvent system $(1: 1 ; 30 \mathrm{~mL})$ at room temperature for 20 minutes. The reaction mixture was then filtered and to the clear filtrate, a solution of the appropriate aryl sulfonyl chloride (0.012 mol) in acetone/water system (1:1; $5 \mathrm{~mL})$ was added dropwise over a period of 20 minutes. The resulted mixture was further stirred for 24 hours at room temperature. The separated solid was then filtered, washed with cold water, dried and crystallized from ethanol. The yield percentages, melting points, molecular formulae and micro-analytical data for (compounds 3a-r) are shown in table 1.

5-Benzylidene-3-phenylsulfonylimidazolidine-2,4-diones (3a): $\mathrm{H}^{1} \mathrm{NMR}\left(\mathrm{DMSO}-\mathrm{d}_{6}\right) ; \delta 6.61(\mathrm{~s}, 1 \mathrm{H}$, $=\mathrm{CH}-$ ), 7.33-7.39 (m, 3H, Ar-H), 7.60 (d, 2H, J = 6.9 Hz, 2H, Ar-H), 7.68-7.85 (m, 3H, Ar-H), 8.07 (d, $2 \mathrm{H}, J=7.6 \mathrm{~Hz}, \mathrm{Ar}-\mathrm{H}$ ), 11.33 (s, $1 \mathrm{H}, \mathrm{NH}$, exchangeable with $\mathrm{D}_{2} \mathrm{O}$ ); $\mathrm{C}^{13}$ NMR (DMSO- $d_{6}$ ): $\delta$ 112.61, 124.92, 128.38, 129.24, 129.63, 130.12, 130.24, 132.60, 135.66, 138.04, 149.85, 160.28.

5-Benzylidene-3-(4-chlorophenylsulfonyl)imidazolidine-2,4-diones (3b): $\mathrm{IR}\left(\mathrm{KBr}, \mathrm{cm}^{-1}\right)$ v: 3222 (NH), 1783, $1745(\mathrm{C}=\mathrm{O}), 1318,1189(\mathrm{O}=\mathrm{S}=\mathrm{O})$; $\mathrm{H}^{1}$ NMR (DMSO-d $)$ ) $\delta: 6.60$ (s, 1H, =CH-), 7.32-7.41 (m, 3H, Ar-H), 7.59 (d, 2H, $J=6.9 \mathrm{~Hz}, \mathrm{Ar}-\mathrm{H}), 7.74$ (d, 2H, $J=8.4 \mathrm{~Hz}, \mathrm{Ar}-\mathrm{H}), 8.06$ (d, 2H, $J=8.4 \mathrm{~Hz}$, Ar-H), 11.35 (s, 1H, NH exchangeable with $\mathrm{D}_{2} \mathrm{O}$ ); $\mathrm{C}^{13}$ NMR (DMSO-d $d_{6}$ ); $\delta 112.60,124.97,129.22$, 129.60, 130.17, 130.21, 130.49, 132.59, 136.67, 140.71, 149.79, 160.20.

5-Benzylidene-3-(4-methylphenylsulfonyl)imidazolidine-2,4-diones (3c): IR ( $\left.\mathrm{KBr}, \mathrm{cm}^{-1}\right)$ v: 3221 (NH), 1784, $1752(\mathrm{C}=\mathrm{O}), 1318,1184(\mathrm{O}=\mathrm{S}=\mathrm{O}) ; \mathrm{H}^{1} \mathrm{NMR}\left(\mathrm{DMSO}-d_{6}\right) \delta 2.48\left(\mathrm{~s}, 3 \mathrm{H}, \mathrm{CH}_{3}\right), 6.62(\mathrm{~s}, 1 \mathrm{H}$, $=\mathrm{CH}-), 6.62(\mathrm{~s}, 1 \mathrm{H},=\mathrm{CH}-), 7.40-7.65(\mathrm{~m}, 7 \mathrm{H}, \mathrm{Ar}-\mathrm{H}), 7.96$ (d, 2H, $J=7.8 \mathrm{~Hz}, \mathrm{Ar}-\mathrm{H}), 11.30$ (s, 1H, NH exchangeable with $\mathrm{D}_{2} \mathrm{O}$ ). 
5-(4-Bromobenzylidene)-3-phenylsulfonylimidazolidine-2,4-diones (3d): $\mathrm{IR}\left(\mathrm{KBr}, \mathrm{cm}^{-1}\right) \mathrm{v}: 3226$ (NH), 1792, 1736 (C=O), 1383, $1190(\mathrm{O}=\mathrm{S}=\mathrm{O}) ; \mathrm{H}^{1} \mathrm{NMR}\left(\mathrm{DMSO}-\mathrm{d}_{6}\right) \delta$ : 6.60 (s, 1H, =CH-), 7.32-7.42 (m, 3H, Ar-H), 7.60 (d, 2H, $J=7.2 \mathrm{~Hz}, \mathrm{Ar}-\mathrm{H}), 7.77$ (d, 2H, $J=8.4 \mathrm{~Hz}, \mathrm{Ar}-\mathrm{H}$ ), 8.05 (d, $2 \mathrm{H}, J=8.7 \mathrm{~Hz}$, Ar-H), 11.33 (s, 1H, NH, exchangeable with $\mathrm{D}_{2} \mathrm{O}$ ); $\mathrm{C}^{13}$ NMR (DMSO-d $)$; $\delta$ 112.53, 125.04, 129.26, 129.63, 130.21, 130.51, 132.61, 136.68, 140.69, 149.79, 160.22.

5-(4-Bromobenzylidene)-3-(4-chlorophenylsulfonyl)imidazolidine-2,4-diones $\quad$ (3e): $\quad H^{1} \quad$ NMR $\left(\mathrm{DMSO}_{\mathrm{C}}\right.$ ) $\delta: 6.60$ (s, 1H, =CH-), 7.80 (d, 2H, $\left.J=8.1 \mathrm{~Hz}, \mathrm{Ar}-\mathrm{H}\right), 7.90$ (d, 2H, $\left.J=7.9 \mathrm{~Hz}, \mathrm{Ar}-\mathrm{H}\right), 7.98$ (d, 2H, $J=8.1 \mathrm{~Hz}, \mathrm{Ar}-\mathrm{H}), 8.15$ (d, 2H, $J=8.0 \mathrm{~Hz}, \mathrm{Ar}-\mathrm{H}), 11.36$ (s, $1 \mathrm{H}, \mathrm{NH}$, exchangeable with $\mathrm{D}_{2} \mathrm{O}$ ).

5-(4-Bromobenzylidene)-3-(4-methylphenylsulfonyl)imidazolidine-2,4-diones (3f): IR (KBr, $\left.\mathrm{cm}^{-1}\right)$ v: $3228(\mathrm{NH})$, 1793, $1736(\mathrm{C}=\mathrm{O})$, 1383, $1189(\mathrm{O}=\mathrm{S}=\mathrm{O}) ; \mathrm{H}^{1}$ NMR (DMSO-d 6 ) $\delta: 2.45$ (s, 3H, $\left.\mathrm{CH}_{3}\right), 6.59$ (s, 1H, =CH-), 7.51 (d, 2H, $J=8.5 \mathrm{~Hz}, \mathrm{Ar}-\mathrm{H}), 7.74$ (d, 2H, $J=7.7 \mathrm{~Hz}, \mathrm{Ar}-\mathrm{H}), 7.94$ (d, 2H, $J=8.5 \mathrm{~Hz}$, Ar-H), 8.01 (d, 2H, $J=7.9 \mathrm{~Hz}$, Ar-H), 11.34 (s, 1H, NH, exchangeable with $\mathrm{D}_{2} \mathrm{O}$ ).

5-(4-Chlorobenzylidene)-3-phenylsulfonylimidazolidine-2,4-diones (3g): IR ( $\left.\mathrm{KBr}, \mathrm{cm}^{-1}\right)$; v: 3224 (NH), 1794, $1736(\mathrm{C}=\mathrm{O}), 1384,1190(\mathrm{O}=\mathrm{S}=\mathrm{O}) ; \mathrm{H}^{1}$ NMR (DMSO-d $)$ ): $6.63(\mathrm{~s}, 1 \mathrm{H},=\mathrm{CH}-), 7.48(\mathrm{~d}$, 2H, $J=7.7 \mathrm{~Hz}, \mathrm{Ar}-\mathrm{H}), 7.64$ (d, 2H, $J=7.8 \mathrm{~Hz}, \mathrm{Ar}-\mathrm{H}), 7.70-7.94$ (m, 5H, Ar-H), 11.33 (s, 1H, NH, exchangeable with $\mathrm{D}_{2} \mathrm{O}$ ).

5-(4-Chlorobenzylidene)-3-(4-chlorophenylsulfonyl)imidazolidine-2,4-diones $\quad$ (3h): $\quad H^{1} \quad$ NMR $\left(\mathrm{DMSO}-\mathrm{d}_{6}\right) \delta: 6.60$ (s, 1H, =CH-), 7.46 (d, 2H, $\left.J=8.4 \mathrm{~Hz}, \mathrm{Ar}-\mathrm{H}\right), 7.63$ (d, 2H, $\left.J=8.4 \mathrm{~Hz}, \mathrm{Ar}-\mathrm{H}\right), 7.78$ (d, $2 \mathrm{H}, J=8.7 \mathrm{~Hz}$, Ar-H), 8.05 (d, $2 \mathrm{H}, J=8.5 \mathrm{~Hz}, \mathrm{Ar}-\mathrm{H}), 11.38\left(\mathrm{~s}, 1 \mathrm{H}, \mathrm{NH}\right.$, exchangeable with $\mathrm{D}_{2} \mathrm{O}$ );

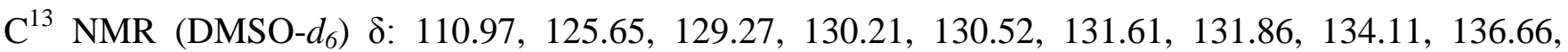
140.70, 149.80, 160.13 .

5-(4-Chlorobenzylidene)-3-(4-methylphenylsulfonyl)imidazolidine-2,4-diones $\quad$ (3i): $\quad H^{1} \quad$ NMR $\left.\left(\mathrm{DMSO}_{-}\right)_{6}\right) \delta: 2.43$ (s, 3H, $\left.\mathrm{CH}_{3}\right), 6.60$ (s, 1H, =CH-), 7.46 (d, 2H, $\left.J=8.6 \mathrm{~Hz}, \operatorname{Ar}-\mathrm{H}\right), 7.52(\mathrm{~d}, 2 \mathrm{H}, J=$ 
$8.4 \mathrm{~Hz}, \mathrm{Ar}-\mathrm{H}$ ), 7.65 (d, 2H, $J=8.0 \mathrm{~Hz}, \mathrm{Ar}-\mathrm{H}), 7.94$ (d, 2H, $J=8.1 \mathrm{~Hz}, \mathrm{Ar}-\mathrm{H}), 11.32$ (s, 1H, NH, exchangeable with $\mathrm{D}_{2} \mathrm{O}$ ).

5-(3-Nitrobenzylidene)-3-phenylsulfonylimidazolidine-2,4-diones (3j): $\mathrm{H}^{1} \mathrm{NMR}\left(\mathrm{DMSO}-d_{6}\right) \delta: 6.76$ (s, 1H, =CH-), 7.55-8.06 (m, 7H, Ar-H), 8.18 (d, 1H, J = 3.3 Hz, Ar-H), 8.40 (s, 1H, Ar-H), 11.60 (br.s, $1 \mathrm{H}, \mathrm{NH}$, exchangeable with $\mathrm{D}_{2} \mathrm{O}$ ).

5-(3-Nitrobenzylidene)-3-(4-chlorophenylsulfonyl)imidazolidine-2,4-diones (3k): $\mathrm{IR}\left(\mathrm{KBr}, \mathrm{cm}^{-1}\right) \mathrm{v}$ : $3429(\mathrm{NH}), 1740,1693(\mathrm{C}=\mathrm{O}), 1390,1166(\mathrm{O}=\mathrm{S}=\mathrm{O})$; $\mathrm{H}^{1} \mathrm{NMR}\left(\mathrm{DMSO}-d_{6}\right) \delta: 6.05$ (s, 1H, =CH-), 7.52 (dd, 1H, $J=3.9,7.5 \mathrm{~Hz}$, Ar-H), 7.72 (d, 2H, $J=7.9 \mathrm{~Hz}, \mathrm{Ar}-\mathrm{H}), 7.92$ (d, 1H, $J=4.1 \mathrm{~Hz}, \mathrm{Ar}-\mathrm{H}), 7.98$ (d, 2H, $J=7.8 \mathrm{~Hz}, \mathrm{Ar}-\mathrm{H}), 8.15$ (d, 1H, $J=3.3 \mathrm{~Hz}, \mathrm{Ar}-\mathrm{H}$ ), 8.43 (s, 1H, Ar-H), 11.42 (br., s, 1H, NH, exchangeable with $\mathrm{D}_{2} \mathrm{O}$ ).

5-(3-Nitrobenzylidene)-3-(4-methylphenylsulfonyl)imidazolidine-2,4-diones (3I): $\mathrm{H}^{1}$ NMR (DMSO$\left.d_{6}\right) \delta: 2.47$ (s, 3H, CH3), 6.77 (s, 1H, =CH-), 7.51 (dd, 1H, $\left.J=3.1,7.7 \mathrm{~Hz}, \mathrm{Ar}-\mathrm{H}\right), 7.61$ (d, 2H, $J=8.1$ Hz, Ar-H), 7.89 (d, 1H, $J=3.2 \mathrm{~Hz}, \mathrm{Ar}-\mathrm{H}), 7.96$ (d, 2H, $J=7.9 \mathrm{~Hz}, \mathrm{Ar}-\mathrm{H}), 8.16$ (d, $1 \mathrm{H}, J=4.1 \mathrm{~Hz}, \mathrm{Ar}-$ H), 8.44 (s, 1H, Ar-H), 11.61 (br., s, $1 \mathrm{H}, \mathrm{NH}$ exchangeable with $\mathrm{D}_{2} \mathrm{O}$ ).

5-(4-Methoxybenzylidene)-3-phenylsulfonylimidazolidine-2,4-diones (3m): $\mathrm{H}^{1} \mathrm{NMR}$ (DMSO- $\left.d_{6}\right) \delta$ : $3.78\left(\mathrm{~s}, 3 \mathrm{H}, \mathrm{OCH}_{3}\right), 6.59$ (s, 1H, =CH-), 6.96 (d, 2H, $\left.J=8.1 \mathrm{~Hz}, \operatorname{Ar}-\mathrm{H}\right), 7.59$ (d, 2H, $\left.J=8.4 \mathrm{~Hz}, \operatorname{Ar}-\mathrm{H}\right)$, 7.67-7.84 (m, 3H, Ar-H), 8.06 (d, 2H, $J=7.5 \mathrm{~Hz}, \mathrm{Ar}-\mathrm{H}), 11.22$ (s, 1H, NH, exchangeable with $\mathrm{D}_{2} \mathrm{O}$ ); $\mathrm{C}^{13}$ NMR (DMSO- $\left.d_{6}\right) \delta:$ 55.78, 113.23, 114.82, 122.80, 125.08, 128.30, 130.12, 132.19, 135.61, 138.12, 149.79, 160.34, 160.59 .

5-(4-Methoxybenzylidene)-3-(4-chlorophenylsulfonyl)imidazolidine-2,4-diones (3n): $\mathrm{IR}\left(\mathrm{KBr}, \mathrm{cm}^{-1}\right)$ v: $3231(\mathrm{NH})$, 1778, $1740(\mathrm{C}=\mathrm{O}), 1319,1186(\mathrm{O}=\mathrm{S}=\mathrm{O})$; $\mathrm{H}^{1} \mathrm{NMR}\left(\mathrm{DMSO}-d_{6}\right) \delta: 3.80\left(\mathrm{~s}, 3 \mathrm{H}, \mathrm{OCH}_{3}\right)$, $6.60(\mathrm{~s}, 1 \mathrm{H},=\mathrm{CH}-), 6.98(\mathrm{~d}, 2 \mathrm{H}, J=7.9 \mathrm{~Hz}, \operatorname{Ar}-\mathrm{H}), 7.62(\mathrm{~d}, 2 \mathrm{H}, J=8.5 \mathrm{~Hz}, \mathrm{Ar}-\mathrm{H}), 7.80(\mathrm{~d}, 2 \mathrm{H}, J=7.9$ Hz, Ar-H), 8.06 (d, 2H, $J=8.2$ Ar-H), 11.23 (s, 1H, NH, exchangeable with $\mathrm{D}_{2} \mathrm{O}$ ). 
$\left(\mathrm{DMSO}_{6}\right) \delta: 2.43$ (s, 3H, $\mathrm{CH}_{3}$ ), 3.80 (s, 3H, $\left.\mathrm{OCH}_{3}\right), 6.60$ (s, $\left.1 \mathrm{H},=\mathrm{CH}-\right), 6.98(\mathrm{~d}, 2 \mathrm{H}, J=7.8 \mathrm{~Hz}, \mathrm{Ar}-$ H), 7.52 (d, 2H, $J=8.4 \mathrm{~Hz}$, Ar-H), 7.61 (d, 2H, $J=8.2 \mathrm{~Hz}, \mathrm{Ar}-\mathrm{H}), 7.94$ (d, 2H, $J=8.0 \mathrm{~Hz}, \mathrm{Ar}-\mathrm{H}), 11.20$ (s, $1 \mathrm{H}, \mathrm{NH}$, exchangeable with $\mathrm{D}_{2} \mathrm{O}$ ).

5-(4-(N,N-Dimethylamino)benzylidene)-3-phenylsulfonylimidazolidine-2,4-diones $\quad(3 p): \quad H^{1} \mathrm{NMR}$ $\left(\mathrm{DMSO}_{6}\right) \delta: 2.97$ (s, 6H, N($\left.\left(\mathrm{CH}_{3}\right)_{2}\right), 6.55$ (s, 1H, =CH-), 6.68 (d, 2H, $\left.J=8.7 \mathrm{~Hz}, \mathrm{Ar}-\mathrm{H}\right), 7.49$ (d, 2H, $J$ $=8.7 \mathrm{~Hz}, \mathrm{Ar}-\mathrm{H}), 7.67-7.84(\mathrm{~m}, 3 \mathrm{H}, \mathrm{Ar}-\mathrm{H}), 8.04$ (d, 2H, $J=8.0 \mathrm{~Hz}, \mathrm{Ar}-\mathrm{H}), 11.04(\mathrm{~s}, 1 \mathrm{H}, \mathrm{NH}$, exchangeable with $\mathrm{D}_{2} \mathrm{O}$ ); $\mathrm{C}^{13}$ NMR $\left(\mathrm{DMSO}_{-} \mathrm{d}_{6}\right) \delta$ : 112.25, 115.27, 119.66, 119.92, 128.19, 130.13, 132.24, 135.55, 138.29, 149.60, 151.29.

5-(4-(N,N-Dimethylamino)benzylidene)-3-(4-chlorophenylsulfonyl)imidazolidine-2,4-diones

IR (KBr, cm $\left.{ }^{-1}\right)$; v: $3211(\mathrm{NH})$, 1729, $1711(\mathrm{C}=\mathrm{O}), 1380,1172(\mathrm{O}=\mathrm{S}=\mathrm{O})$; H1 NMR (DMSO-d6) $\delta 2.99$ (s, 6H, N(CH$\left.)_{2}\right), 6.57$ (s, 1H, =CH-), $6.71(\mathrm{~d}, 2 \mathrm{H}, J=8.5 \mathrm{~Hz}, \operatorname{Ar}-\mathrm{H}), 7.52$ (d, 2H, $\left.J=7.9 \mathrm{~Hz}, \operatorname{Ar}-\mathrm{H}\right)$, 7.79 (d, 2H, $J=8.2 \mathrm{~Hz}$, Ar-H), 8.06 (d, 2H, $J=8.4 \mathrm{~Hz}$, Ar-H), 11.06 (s, 1H, NH exchangeable, with $\left.\mathrm{D}_{2} \mathrm{O}\right)$.

5-(4-(N,N-Dimethylamino)benzylidene)-3-(4-methylphenylsulfonyl)imidazolidine-2,4-diones (3r): $\mathrm{H}^{1} \mathrm{NMR}\left(\mathrm{DMSO}-d_{6}\right) \delta: 2.43$ (s, 3H, Ar- $\left.\mathrm{CH}_{3}\right), 2.99$ (s, 6H, N(CH$\left.)_{2}\right), 6.56$ (s, 1H, =CH-), 6.70 (d, 2H, $J$ = $7.9 \mathrm{~Hz}, \mathrm{Ar}-\mathrm{H}), 7.51$ (d, 4H, $J=7.5 \mathrm{~Hz}, \mathrm{Ar}-\mathrm{H}), 7.94$ (d, 2H, $J=7.8 \mathrm{~Hz}, \mathrm{Ar}-\mathrm{H}), 11.03$ (s, 1H, NH, exchangeable with $\left.\mathrm{D}_{2} \mathrm{O}\right)$.

\subsubsection{General procedure for the synthesis of compounds $6 a-1$.}

A mixture of compounds 5a-d $(0.01 \mathrm{~mol})$ and $\mathrm{K}_{2} \mathrm{CO}_{3}(0.69 \mathrm{~g}, 0.005 \mathrm{~mol})$ was stirred in acetone/water mixture $(1: 1 ; 30 \mathrm{~mL})$ at room temperature for 10 minutes. To the resulted solution; the appropriate arylsulfonyl chloride $(0.012 \mathrm{~mol})$ in acetone/water system $(1: 1 ; 5 \mathrm{~mL})$ was added dropwise over a period 
of 20 minutes. The reaction mixture was further stirred at room temperature for 24 hours. The separated solid was then filtered, washed with cold water, dried and crystallized from the appropriate solvent. The yield percentages, melting points, molecular formulae and micro-analytical data for compounds 6a-I are shown in table 2:

1-Phenyl-3-phenylsulfonylpyrimidine-2,4,6-(1H,3H,5H)-triones $(\mathbf{6 a})$ : IR $\left(\mathrm{KBr}, \mathrm{cm}^{-1}\right)$ v: 1728, 1655 (C=O), 1374, $1165(\mathrm{O}=\mathrm{S}=\mathrm{O})$; H1 NMR (DMSO-d6); $\delta 5.36\left(\mathrm{~s}, 2 \mathrm{H}, \mathrm{CH}_{2}\right)$, 7.32-7.86 (m, 8H, Ar-H), 8.06 (d, 2H, $J=7.9 \mathrm{~Hz}$, Ar-H); C ${ }^{13}$ NMR (DMSO-d $\left.)_{6}\right) \delta: 112.55,124.97,128.37,129.26,129.65,130.13$, 130.24, 132.61, 135.67, 138.02, 149.86, 160.30 .

1-Phenyl-3-(4-chlorophenylsulfonyl)pyrimidine-2,4,6-(1H,3H,5H)-triones (6b): $\mathrm{H}^{1} \mathrm{NMR}\left(\mathrm{DMSO}-\mathrm{d}_{6}\right)$

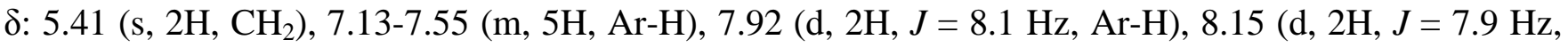
Ar-H); MS m/z (\%): 380 (5.15, $\mathrm{M}^{+}+$2), 378 (13.25, $\mathrm{M}^{+}$), 204 (14.76), 175 (89.07), 119 (100), 111 (62.28), 77 (25.94).

1-Phenyl-3-(4-methylphenylsulfonyl)pyrimidine-2,4,6-(1H,3H,5H)-triones (6c): $\mathrm{H}^{1} \mathrm{NMR}$ (DMSO$\left.d_{6}\right) \delta: 2.51\left(\mathrm{~s}, 3 \mathrm{H}, \mathrm{CH}_{3}\right), 5.35$ (s, 2H, $\left.\mathrm{CH}_{2}\right), 7.10-7.50$ (m, 5H, Ar-H), 7.61 (d, 2H, J = 7.6 Hz, Ar-H), 8.01 (d, 2H, $J=8.2 \mathrm{~Hz}, \operatorname{Ar}-\mathrm{H}) ; \mathrm{MS}$ m/z (\%): 359 (3.22, $\left.\mathrm{M}^{+}+1\right), 358$ (5.23, $\left.\mathrm{M}^{+}\right), 204$ (7.94), 155 (37.09), 119 (45.08), 91 (100), 77 (26.53).

1-(4-Chlorophenyl)-3-phenylsulfonylpyrimidine-2,4,6-(1H,3H,5H)-triones (6d): $\mathrm{H}^{1} \mathrm{NMR}$ (DMSO$\left.d_{6}\right) \delta: 5.35\left(\mathrm{~s}, 2 \mathrm{H}, \mathrm{CH}_{2}\right), 7.32(\mathrm{~d}, 2 \mathrm{H}, J=8.1 \mathrm{~Hz}, \mathrm{Ar}-\mathrm{H}), 7.54(\mathrm{~d}, 2 \mathrm{H}, J=8.0 \mathrm{~Hz}, \mathrm{Ar}-\mathrm{H}), 7.65-8.10(\mathrm{~m}$, 5H, Ar-H).

1-(4-Chlorophenyl)-3-(4-chlorophenylsulfonyl)pyrimidine-2,4,6-(1H,3H,5H)-triones (6e): $\mathrm{H}^{1} \mathrm{NMR}$ $\left.\left(\mathrm{DMSO}_{6}\right)_{6}\right)$ : 5.39 (s, 2H, $\mathrm{CH}_{2}$ ), 7.30 (d, 2H, $\left.J=7.9 \mathrm{~Hz}, \mathrm{Ar}-\mathrm{H}\right), 7.53$ (d, 2H, $\left.J=8.0 \mathrm{~Hz}, \mathrm{Ar}-\mathrm{H}\right), 7.90$ (d, 2H, $J=7.7 \mathrm{~Hz}, \mathrm{Ar}-\mathrm{H}), 8.15$ (d, 2H, $J=8.0 \mathrm{Ar}-\mathrm{H})$. 
1-(4-Chlorophenyl)-3-(4-methylphenylsulfonyl)pyrimidine-2,4,6-(1H,3H,5H)-triones (6f): $\mathrm{IR}(\mathrm{KBr}$, $\left.\mathrm{cm}^{-1}\right)$; v: 1736, $1650(\mathrm{C}=\mathrm{O}), 1381,1170(\mathrm{O}=\mathrm{S}=\mathrm{O}) ; \mathrm{H}^{1} \mathrm{NMR}\left(\mathrm{DMSO}-d_{6}\right) ; \delta 2.49\left(\mathrm{~s}, 3 \mathrm{H}, \mathrm{CH}_{3}\right), 5.35(\mathrm{~s}$, 2H, $\mathrm{CH}_{2}$ ), 7.30 (d, 2H, $\left.J=7.9 \mathrm{~Hz}, \mathrm{Ar}-\mathrm{H}\right), 7.52$ (d, 2H, $\left.J=7.9 \mathrm{~Hz}, \mathrm{Ar}-\mathrm{H}\right), 7.61$ (d, 2H, $J=8.3 \mathrm{~Hz}$, ArH), 8.02 (d, 2H, $J=8.5 \mathrm{~Hz}$, Ar-H).

1-(4-Methylphenyl)-3-phenylsulfonylpyrimidine-2,4,6-(1H,3H,5H)-triones (6g): $\mathrm{H}^{1} \mathrm{NMR}$ (DMSO$\left.d_{6}\right) \delta: 2.34\left(\mathrm{~s}, 3 \mathrm{H}, \mathrm{CH}_{3}\right), 5.41\left(\mathrm{~s}, 2 \mathrm{H}, \mathrm{CH}_{2}\right), 7.12$ (d, $\left.2 \mathrm{H}, J=8.0 \mathrm{~Hz}, \mathrm{Ar}-\mathrm{H}\right), 7.25$ (d, $2 \mathrm{H}, J=7.8 \mathrm{~Hz}, \mathrm{Ar}-$ H), 7.63-8.01 (m, 5H, Ar-H).

1-(4-Methylphenyl)-3-(4-chlorophenylsulfonyl)pyrimidine-2,4,6-(1H,3H,5H)-triones (6h): $\mathrm{IR}(\mathrm{KBr}$, $\left.\mathrm{cm}^{-1}\right)$ v: 1730, $1662(\mathrm{C}=\mathrm{O}), 1381,1168(\mathrm{O}=\mathrm{S}=\mathrm{O}) ; \mathrm{H}^{1} \mathrm{NMR}\left(\mathrm{DMSO}-\mathrm{d}_{6}\right) \delta: 2.35\left(\mathrm{~s}, 3 \mathrm{H}, \mathrm{CH}_{3}\right), 5.43(\mathrm{~s}$, 2H, $\mathrm{CH}_{2}$ ), 7.11 (d, 2H, $\left.J=8.0 \mathrm{~Hz}, \mathrm{Ar}-\mathrm{H}\right), 7.25$ (d, 2H, $\left.J=8.0 \mathrm{~Hz}, \mathrm{Ar}-\mathrm{H}\right), 7.90$ (d, 2H, $J=7.8 \mathrm{~Hz}, \mathrm{Ar}-$ H), 8.15 (d, 2H, $J=8.0 \mathrm{~Hz}, \mathrm{Ar}-\mathrm{H})$.

1-(4-Methylphenyl)-3-(4-methylphenylsulfonyl)pyrimidine-2,4,6-(1H,3H,5H)-triones $(6 \mathbf{i}): \mathrm{H}^{1} \mathrm{NMR}$ $\left(\mathrm{DMSO}_{-} \mathrm{d}_{6}\right) \delta: 2.35$ (s, 3H, $\mathrm{CH}_{3}$ ), 2.52 (s, 3H, $\mathrm{CH}_{3}$ ), 5.40 (s, 2H, $\mathrm{CH}_{2}$ ), 7.11 (d, $\left.2 \mathrm{H}, J=7.6 \mathrm{~Hz}, \mathrm{Ar}-\mathrm{H}\right)$, 7.26 (d, 2H, $J=7.6 \mathrm{~Hz}, \mathrm{Ar}-\mathrm{H}), 7.60$ (d, 2H, $J=8.3 \mathrm{~Hz}, \mathrm{Ar}-\mathrm{H}), 7.97$ (d, 2H, $J=8.0 \mathrm{~Hz}, \mathrm{Ar}-\mathrm{H})$.

1-(4-Methoxyphenyl)-3-phenylsulfonylpyrimidine-2,4,6-(1H,3H,5H)-triones (6j): $\mathrm{H}^{1} \mathrm{NMR}$ (DMSO$\left.d_{6}\right) \delta: 3.79\left(\mathrm{~s}, 3 \mathrm{H}, \mathrm{OCH}_{3}\right), 5.34$ (s, 2H, $\left.\mathrm{CH}_{2}\right), 7.00$ (d, 2H, $\left.J=7.8 \mathrm{~Hz}, \mathrm{Ar}-\mathrm{H}\right), 7.15$ (d, 2H, $J=7.8 \mathrm{~Hz}, \mathrm{Ar}-$ H), 7.68-8.04 (m, 5H, Ar-H).

1-(4-Methoxyphenyl)-3-(4-chlorophenylsulfonyl)pyrimidine-2,4,6-(1H,3H,5H)-triones

(6k):

$\mathrm{H}^{1} \mathrm{NMR}\left(\mathrm{DMSO}-\mathrm{d}_{6}\right.$ ) $\delta: 3.79$ (s, 3H, $\mathrm{OCH}_{3}$ ), 5.32 (s, 2H, $\mathrm{CH}_{2}$ ), 6.96 (d, 2H, $\left.J=8.7 \mathrm{~Hz}, \mathrm{Ar}-\mathrm{H}\right), 7.61$ (d, 2H, $J=8.4 \mathrm{~Hz}, \mathrm{Ar}-\mathrm{H}), 7.78(\mathrm{~d}, 2 \mathrm{H}, J=8.4 \mathrm{~Hz}, \mathrm{Ar}-\mathrm{H}), 8.04$ (d, 2H, $J=8.4 \mathrm{~Hz}, \mathrm{Ar}-\mathrm{H})$; $\mathrm{C}^{13} \mathrm{NMR}$ (DMSO-d $\left.)_{6}\right)$ : 114.77, 115.69, 122.96, 128.04, 128.57, 129.28, 129.57, 135.64, 136.23, 140.28, 150.68, 162.69. 
1-(4-Methoxyphenyl)-3-(4-methylphenylsulfonyl)pyrimidine-2,4,6-(1H,3H,5H)-triones

(6l): IR

$\left(\mathrm{KBr}, \mathrm{cm}^{-1}\right)$ v: 1729, $1660(\mathrm{C}=\mathrm{O}), 1370,1181(\mathrm{O}=\mathrm{S}=\mathrm{O}) ; \mathrm{H}^{1} \mathrm{NMR}\left(\mathrm{DMSO}-d_{6}\right) \delta: 2.42\left(\mathrm{~s}, 3 \mathrm{H}, \mathrm{CH}_{3}\right), 3.79$ (s, 3H, $\mathrm{OCH}_{3}$ ), 5.32 (s, 2H, CH 2 ), 6.96 (d, 2H, $\left.J=8.7 \mathrm{~Hz}, \mathrm{Ar}-\mathrm{H}\right), 7.50$ (d, 2H, $J=8.0 \mathrm{~Hz}, \mathrm{Ar}-\mathrm{H}$ ), 7.60 (d, 2H, $J=8.4 \mathrm{~Hz}, \mathrm{Ar}-\mathrm{H}), 7.92(\mathrm{~d}, 2 \mathrm{H}, J=8.1 \mathrm{~Hz}, \mathrm{Ar}-\mathrm{H})$.

\subsubsection{General procedure for the synthesis of compounds $8 a-l$.}

The appropriate compound 3a-d $(0.01 \mathrm{~mol})$ was added in one portion to a solution of metallic sodium (0.345 g, $0.015 \mathrm{~mol})$ in tert-butanol (30 mL). The reaction mixture was heated under reflux for 6 hours. The precipitated salt was filtered while hot, washed with hot tert-butanol (50 mL) and dried. To a suspension of the separated salt $(0.005 \mathrm{~mol})$ in tert-butanol $(20 \mathrm{~mL})$; the appropriate aryl sulfonyl chloride $(0.006 \mathrm{~mol})$ in tert-butanol $(5 \mathrm{~mL})$ was added dropwise while heating and stirring. The reaction mixture was heated under reflux for 12 hours. The solvent was evaporated under reduced pressure, and the obtained solid was stirred with aqueous $\mathrm{NaOH}$ solution $(5 \%, 100 \mathrm{~mL}$ ) for 30 minutes. The separated solid was filtered, washed with water, dried and crystallized from ethanol. The yield percentages, melting points, molecular formulae and micro-analytical data (for compounds 8a-l are shown in table 3.

3-Phenyl-1-phenylsulfonylquinazoline-2,4(1H,3H)-diones (8a): IR ( $\left.\mathrm{KBr}, \mathrm{cm}^{-1}\right)$ v: 1727, $1679(\mathrm{C}=\mathrm{O})$, 1378, $1165(\mathrm{O}=\mathrm{S}=\mathrm{O}) ; \mathrm{H}^{1} \mathrm{NMR}\left(\mathrm{CDCl}_{3}\right) \delta:$ 7.25-8.23 (m, 14H, Ar-H); $\mathrm{C}^{13} \mathrm{NMR}\left(\mathrm{DMSO}-d_{6}\right) \delta:$ 118.43, 120.12, 126.13, 128.55, 128.74, 129.20, 129.49, 130.02, 134.97, 135.27, 135.32, 136.89, 139.03, 149.00, 161.36.

3-Phenyl-1-(4-chlorophenylsulfonyl)quinazoline-2,4(1H,3H)-diones $(\mathbf{8 b})$ : IR $\left(\mathrm{KBr}, \mathrm{cm}^{-1}\right)$ v: 1729, $1681(\mathrm{C}=\mathrm{O}), 1377,1169(\mathrm{O}=\mathrm{S}=\mathrm{O}) ; \mathrm{H}^{1} \mathrm{NMR}\left(\mathrm{CDCl}_{3}\right)$ 8: 7.24-8.22 (m, 13H, Ar-H).

3-Phenyl-1-(4-methylphenylsulfonyl)quinazoline-2,4(1H,3H)-diones (8c): IR ( $\left.\mathrm{KBr}, \mathrm{cm}^{-1}\right)$ v: 1729, $1679(\mathrm{C}=\mathrm{O}), 1377,1168(\mathrm{O}=\mathrm{S}=\mathrm{O}) ; \mathrm{H}^{1} \mathrm{NMR}\left(\mathrm{CDCl}_{3}\right) \delta: 2.47$ (s, 3H, $\left.\mathrm{CH}_{3}\right), 7.09-8.25(\mathrm{~m}, 13 \mathrm{H}, \mathrm{Ar}-\mathrm{H})$. 
3-(4-Chlorophenyl)-1-phenylsulfonylquinazoline-2,4(1H,3H)-diones (8d): IR $\left(\mathrm{KBr}, \mathrm{cm}^{-1}\right)$ v: 1727, $1683(\mathrm{C}=\mathrm{O}), 1377,1170(\mathrm{O}=\mathrm{S}=\mathrm{O}) ; \mathrm{H}^{1} \mathrm{NMR}\left(\mathrm{CDCl}_{3}\right) \delta: 7.06(\mathrm{~d}, 2 \mathrm{H}, J=7.9 \mathrm{~Hz}, \mathrm{Ar}-\mathrm{H}), 7.30(\mathrm{~d}, 2 \mathrm{H}, J=$ 8.2 Hz, Ar-H), 7.44-8.28 (m, 9H, Ar-H).

3-(4-Chlorophenyl)-1-(4-chlorophenylsulfonyl)quinazoline-2,4(1H,3H)-diones (8e): $\mathrm{IR}\left(\mathrm{KBr}, \mathrm{cm}^{-1}\right)$ v: 1729, $1681(\mathrm{C}=\mathrm{O}), 1377,1168(\mathrm{O}=\mathrm{S}=\mathrm{O}) ; \mathrm{H}^{1} \mathrm{NMR}\left(\mathrm{CDCl}_{3}\right) \delta: 7.06(\mathrm{~d}, 2 \mathrm{H}, J=8.4 \mathrm{~Hz}, \mathrm{Ar}-\mathrm{H}), 7.30(\mathrm{~d}$, $2 \mathrm{H}, J=8.5 \mathrm{~Hz}, \mathrm{Ar}-\mathrm{H}), 7.40-8.28$ (m, 8H, Ar-H).

3-(4-Chlorophenyl)-1-(4-methylphenylsulfonyl)quinazoline-2,4(1H,3H)-diones (8f): $\mathrm{IR}\left(\mathrm{KBr}, \mathrm{cm}^{-1}\right)$ v: 1729, $1681(\mathrm{C}=\mathrm{O}), 1376,1170(\mathrm{O}=\mathrm{S}=\mathrm{O}) ; \mathrm{H}^{1} \mathrm{NMR}\left(\mathrm{CDCl}_{3}\right) \delta: 2.47$ (s, 3H, $\left.\mathrm{CH}_{3}\right), 7.07$ (d, $2 \mathrm{H}, J=8.1$ Hz, Ar-H), 7.31 (d, 2H, J = 7.8 Hz, Ar-H), 7.44-8.25 (m, 8H, Ar-H).

3-(4-Methylphenyl)-1-phenylsulfonylquinazoline-2,4(1H,3H)-diones $(8 \mathrm{~g}): \mathrm{IR}\left(\mathrm{KBr}, \mathrm{cm}^{-1}\right)$ v: 1729, $1681(\mathrm{C}=\mathrm{O}), 1381,1174(\mathrm{O}=\mathrm{S}=\mathrm{O}) ; \mathrm{H}^{1} \mathrm{NMR}\left(\mathrm{CDCl}_{3}\right) \delta: 2.40\left(\mathrm{~s}, 3 \mathrm{H}, \mathrm{CH}_{3}\right), 6.99$ (d, $2 \mathrm{H}, \mathrm{J}=8.4 \mathrm{~Hz}, \mathrm{Ar}-$ H), 7.28 (d, 2H, $J=8.0 \mathrm{~Hz}$, Ar-H), 7.44-8.26 (m, 9H, Ar-H).

3-(4-Methylphenyl)-1-(4-chlorophenylsulfonyl)quinazoline-2,4(1H,3H)-diones (8h): $\mathrm{IR}\left(\mathrm{KBr}, \mathrm{cm}^{-1}\right)$ v: 1730, $1684(\mathrm{C}=\mathrm{O}), 1381,1175(\mathrm{O}=\mathrm{S}=\mathrm{O}) ; \mathrm{H}^{1} \mathrm{NMR}\left(\mathrm{CDCl}_{3}\right) \delta: 2.41\left(\mathrm{~s}, 3 \mathrm{H}, \mathrm{CH}_{3}\right), 7.02(\mathrm{~d}, 2 \mathrm{H}, J=7.5$ Hz, Ar-H), 7.30 (d, 2H, J = 7.7 Hz, Ar-H), 7.44-8.28 (m, 8H, Ar-H).

3-(4-Methylphenyl)-1-(4-methylphenylsulfonyl)quinazoline-2,4(1H,3H)-diones (8i): $\mathrm{IR}\left(\mathrm{KBr}, \mathrm{cm}^{-1}\right)$ v: 1730, $1683(\mathrm{C}=\mathrm{O}), 1380,1175(\mathrm{O}=\mathrm{S}=\mathrm{O}) ; \mathrm{H}^{1} \mathrm{NMR}\left(\mathrm{CDCl}_{3}\right) \delta: 2.42\left(\mathrm{~s}, 3 \mathrm{H}, \mathrm{CH}_{3}\right), 2.48\left(\mathrm{~s}, 3 \mathrm{H}, \mathrm{CH}_{3}\right)$, 7.01 (d, 2H, $J=7.8 \mathrm{~Hz}$, Ar-H), 7.28 (d, 2H, $J=7.8 \mathrm{~Hz}$, Ar-H), 7.40-8.24 (m, 8H, Ar-H).

3-(4-Methoxyphenyl)-1-phenylsulfonylquinazoline-2,4(1H,3H)-diones $(\mathbf{8 j})$ : IR $\left(\mathrm{KBr}, \mathrm{cm}^{-1}\right)$ v: 1730, $1684(\mathrm{C}=\mathrm{O}), 1384,1182(\mathrm{O}=\mathrm{S}=\mathrm{O}) ; \mathrm{H}^{1} \mathrm{NMR}\left(\mathrm{CDCl}_{3}\right) \delta: 3.77$ (s, 3H, $\left.\mathrm{OCH}_{3}\right), 6.97(\mathrm{~d}, 2 \mathrm{H}, J=8.9 \mathrm{~Hz}, \mathrm{Ar}-$ H), 7.09 (d, 2H, $J=9.0 \mathrm{~Hz}, \mathrm{Ar}-\mathrm{H}), 7.51$ (t, 1H, $J=7.5 \mathrm{~Hz}, \mathrm{Ar}-\mathrm{H}), 7.67-8.18$ (m, 8H, Ar-H); $\mathrm{C}^{13} \mathrm{NMR}$ 
(DMSO-d $\left.)_{6}\right) \delta:$ 55.83, 114.65, 118.42, 120.09, 126.11, 127.69, 128.51, 128.73, 130.00, 130.16, 134.88, 135.24, 136.77, 139.01, 149.08, 159.63, 161.47.

3-(4-Methoxyphenyl)-1-(4-chlorophenylsulfonyl)quinazoline-2,4(1H,3H)-diones $(8 \mathrm{k})$ : $\mathrm{IR}\left(\mathrm{KBr}, \mathrm{cm}^{-}\right.$ $\left.{ }^{1}\right)$ v: 1732, $1685(\mathrm{C}=\mathrm{O}), 1385,1182(\mathrm{O}=\mathrm{S}=\mathrm{O}) ; \mathrm{H}^{1} \mathrm{NMR}\left(\mathrm{CDCl}_{3}\right) \delta: 3.85\left(\mathrm{~s}, 3 \mathrm{H}, \mathrm{OCH}_{3}\right), 6.98(\mathrm{~d}, 2 \mathrm{H}, J=$ 8.3 Hz, Ar-H), 7.14 (d, 2H, J = 8.5 Hz, Ar-H), 7.40-8.29 (m, 8H, Ar-H).

3-(4-Methoxyphenyl)-1-(4-methylphenylsulfonyl)quinazoline-2,4(1H,3H)-diones (8I): IR ( $\mathrm{KBr}, \mathrm{cm}^{-}$

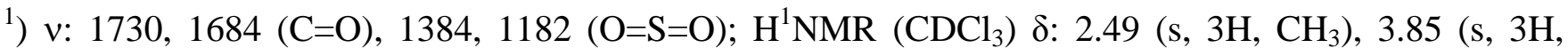
$\mathrm{OCH}_{3}$ ), 6.99 (d, 2H, $\left.J=7.8 \mathrm{~Hz}, \mathrm{Ar}-\mathrm{H}\right), 7.14$ (d, 2H, $\left.J=7.6 \mathrm{~Hz}, \mathrm{Ar}-\mathrm{H}\right), 7.44-8.29$ (m, 8H, Ar-H).

\subsection{Biological evaluation}

The human tumor cell lines of the cancer screening panel are grown in RPMI 1640 medium containing 5\% fetal bovine serum and $2 \mathrm{mM}$ L-glutamine. For a typical screening experiment, cells are inoculated into 96 well microtiter plates in $100 \mu \mathrm{L}$ at plating densities ranging from 5,000 to 40,000 cells/well depending on the doubling time of individual cell lines. After cell inoculation, the microtiter plates are incubated at $37^{\circ} \mathrm{C}, 5 \% \mathrm{CO}_{2}, 95 \%$ air and $100 \%$ relative humidity for $24 \mathrm{~h}$ prior to addition of experimental drugs.

After 24 h, two plates of each cell line are fixed in situ with TCA, to represent a measurement of the cell population for each cell line at the time of drug addition (Tz). Experimental drugs are solubilized in dimethyl sulfoxide at 400-fold the desired final maximum test concentration and stored frozen prior to use. At the time of drug addition, an aliquot of frozen concentrate is thawed and diluted to twice the desired final maximum test concentration with complete medium containing $50 \mu \mathrm{g} / \mathrm{ml}$ gentamicin. Aliquot of $100 \mu \mathrm{l}$ of this drug dilutions was added to the appropriate microtiter wells already containing $100 \mu \mathrm{l}$ of medium, resulting in the required final drug concentrations. 
Following drug addition, the plates are incubated for an additional $48 \mathrm{~h}$ at $37^{\circ} \mathrm{C}, 5 \% \mathrm{CO}_{2}, 95 \%$ air, and $100 \%$ relative humidity. For adherent cells, the assay is terminated by the addition of cold TCA. Cells are fixed in situ by the gentle addition of $50 \mu \mathrm{l}$ of cold $50 \%$ (w/v) TCA (final concentration, $10 \%$ TCA) and incubated for 60 minutes at $4^{\circ} \mathrm{C}$. The supernatant is discarded, and the plates are washed five times with tap water and air dried. Sulforhodamine B (SRB) solution (100 $\mu \mathrm{l})$ at $0.4 \%(\mathrm{w} / \mathrm{v})$ in $1 \%$ acetic acid is added to each well, and plates are incubated for 10 minutes at room temperature. After staining, unbound dye is removed by washing five times with $1 \%$ acetic acid and the plates are air dried. Bound stain is subsequently solubilized with $10 \mathrm{mM}$ trizma base, and the absorbance is read on an automated plate reader at a wavelength of $515 \mathrm{~nm}$. For suspension cells, the methodology is the same except that the assay is terminated by fixing settled cells at the bottom of the wells by gently adding $50 \mu l$ of $80 \%$ TCA (final concentration, $16 \%$ TCA) [55-57].

\subsection{Molecular modeling methods}

\subsubsection{General methodology:}

All molecular modelling calculations were performed using "Molecular Operating Environment (MOE) version 2008.10", Chemical Computing Group Inc., running on "Windows Vista" operating system installed on an Intel core 2du PC with a 1.8 MHz processor and 1000 Mb RAM.

\subsubsection{Alignment of the training set compounds:}

The training set compounds, consisting of nine diarylsulfonylureas of diverse chemical structures including compounds $\mathbf{A}-\mathbf{E}$, in addition to, compounds $\mathbf{3 h}, \mathbf{3 n}, \mathbf{6 b}$ and $\mathbf{8 a}$, were built using the builder interface of the MOE software. The compounds were aligned using the flexible alignment tool of the program adjusting the energy cut off to $10 \mathrm{kcal} / \mathrm{mol}$ and root mean square deviation (RMSD) tolerance to 0.5 . The stochastic conformation search option was used as the method of alignment.

\subsubsection{Target compounds optimization:}


Conformational analyses of the built molecules were performed in a two-step procedure. First, the target compounds were subjected to energy minimization tool using the included MOPAC 7.0. The geometry of the compounds was optimized with the semiemperical AM1 Hamiltonian using Restricted HartreeFock (RHF) and RMS gradient of $0.05 \mathrm{Kcal} / \mathrm{mol}$. Then, the produced model was subjected to the 'Systematic Conformational Search’ of the MOE. All items were set as default with

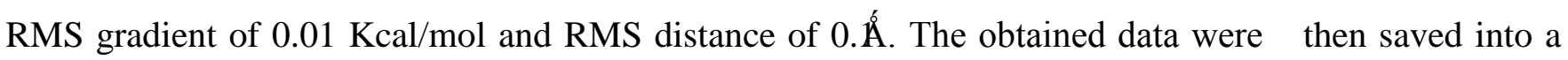
MDB file to be used in the pharmacophore fitting calculations.

\subsubsection{Pharmacophore Building:}

A pharmacophore model was created using the 'Pharmacophore Query Editor'. The aligned training set compounds were used as the template for building the model. The settings of the software parameters were adjusted to: 'Unified' as the scheme of annotation 'Consensus method' for model building. Tolerance was set to $1.2 \AA ̊$ and the threshold was set to 50\%. The produced model was used for testing compound pharmacophore fitting and further calculations.

\subsubsection{Fitting of the target compounds on the built model:}

Using the generated pharmacophore model and the saved conformations of each molecule; the fitting of the target compounds into the model was tested. The root mean square deviation value for each conformer was calculated and the one having the lowest RMSD value was taken for further visual and energy inspection.

\section{Acknowledgments}

The authors would like to express their gratitude and thanks to the National Cancer Institute (NCI), Bethesda Maryland, USA, http://dtp.cancer.gov/ for doing the antitumor testing of the new compounds. Our sincere acknowledgments to Chemical Computing Group Inc, 1010 Sherbrooke Street West, Suite 910, Montreal, H3A 2R7, Canada., for its valuable agreement to use the package of MOE 2008.10 software. 


\section{References}

1. S. Eckhardt, Recent progress in the development of anticancer agents, Curr. Med. Chem. Anti-Canc. Agents 2 (2002) 419-439.

2. C.W. Lee, D.H. Hong, S.B. Han, S.-H. Jong, H.C. Kim, R.L. Fine, S.-H. Lee, H.M. Kim, A novel stereoselective sulfonylurea, 1-[1-(4-aminobenzoyl)-2,3-dihydro-1H-indol-6-sulfonyl]-4-phenyl-imidazolidin2-one, has antitumor efficacy in in-vitro and in-vivo tumor models Biochem. Pharmacol. 64 (2002) 473480.

3. F. Mohamadi, M.M. Spees, G.B. Grindey, Sulfonylureas: a new class of cancer chemotherapeutic agents, J. Med. Chem. 35 (1992) 3012-3016.

4. J.W. Chern, Y.L. Leu, S.S. Wang, R. Jou, C.F. Lee, P.C. Tsou, S.C. Hsu, Y.C. Liaw, H.M. Lin, Synthesis and cytotoxic evaluation of substituted sulfonyl- $N$-hydroxyguanidine derivatives as potential antitumor agents, J. Med. Chem. 40 (1997) 2276-2286.

5. J.E. Toth, G.B. Grindey, W.J. Ehlhardt, J.E. Ray, G.B. Boder, J.R. Bewley, K.K. Klingerman, S.B. Gates, S.M. Rinzel, R.M. Schultz, L.C. Weir, J.F. Worzalla, Sulfonimidamide analogs of oncolytic sulfonylureas, J. Med. Chem. 40 (1997) 1018-1025.

6. J.C. Medina, B. Shan, H. Beckmann, R.P. Farrell, D.L. Clark, R.M. Learned, D. Roche, A. Li, V. Baichwal, C. Case, P.A. Baeuerle, T. Rosen, J.C. Jaen, Novel antineoplastic agents with efficacy against multidrug resistant tumor cells, Bioorg. Med. Chem. Lett. 8 (1998) 2653-2656.

7. J.C. Medina, D. Roche, B. Shan, R.M. Learned, W.P. Frankmoelle, D.L. Clark, T. Rosen, J.C. Jaen, Novel halogenated sulfonamides inhibit the growth of multidrug resistant MCF-7/ADR cancer cells, Bioorg. Med. Chem. Lett. 9 (1999) 1843-1846.

8. A. Mastrolorenzo, A. Scozzafava, C.T. Supuran, 4-Toluenesulfonylureido derivatives of amines, amino acids and dipeptides: a novel class of potential antitumor agents, Eur. J. Pharm. Sci. 11 (2000) 325-332.

9. J.J. Howbert, C.S. Grossman, T.A. Crowell, B.J. Rieder, R.W. Harper, G.B. Grindey, Novel agents effective against solid tumors: the diarylsulfonylureas. Synthesis, activities, and analysis of quantitative structure-activity relationships, J. Med. Chem. 33 (1990) 2393-2407. 
10. C.T. Supuran, F. Briganti, S. Tilli, W.R. Chegwidden, A. Scozzafava, Carbonic anhydrase inhibitors: sulfonamides as antitumor agents? Bioorg. Med. Chem. 9 (2001) 703-714.

11. A. Scozzafava, C.T. Supuran, Carbonic anhydrase inhibitors. Arylsulfonylureido- and arylureidosubstituted aromatic and heterocyclic sulfonamides: towards selective inhibitors of carbonic anhydrase isozyme I, J. Enzyme Inhib. 14 (1999) 343-363.

12. A. Scozzafava, C.T. Supuran, Carbonic anhydrase activators. Part 24. High affinity isozymes I, II and IV activators, derivatives of 4-(4-chlorophenylsulfonylureido-aminoacyl)ethyl-1H-imidazole, Eur. J. Pharm. Sci. 10 (2000) 29-41.

13. A. Casini, A. Scozzafava, A. Mastrolorenzo, C.T. Supuran, Sulfonamides and sulfonylated derivatives as anticancer agents, Curr. Cancer Drug Targets 2 (2002) 55-75.

14. X. Guan, B.N. Hoffman, D.C. McFarland, K.K. Gilkerson, C. Dwivedi, A.K. Erickson, S. Bebensee, J. Pellegrini, Glutathione and mercapturic acid conjugates of sulofenur and their activity against a human colon cancer cell line, Drug Metab. Dispos. 30 (2002) 331-335.

15. B. Forouzesh, C.H. Takimoto, A. Goetz, S. Diab, L.A. Hammond, L. Smetzer, G. Schwartz, R. Gazak, J.T. Callaghan, D.D. Von Hoff, E.K. Rowinsky, A phase I and pharmacokinetic study of ILX-295501, an oral diarylsulfonylurea, on a weekly for 3 weeks every 4-week schedule in patients with advanced solid malignancies, Clin. Cancer Res. 9 (2003) 5540-5549.

16. S.H. Jung, J.S. Song, H.S. Lee, S.U. Choi, C.O. Lee, Synthesis and evaluation of cytotoxic activity of novel arylsulfonylimidazolidinones, Bioorg. Med. Chem. Lett. 6 (1996) 2553-2558.

17. S.H. Jung, J.S. Song, H.S. Lee, S.U. Choi, C.O. Lee, Synthesis and evaluation of cytotoxicity of novel arylsulfonylimidazolidinones containing sulfonylurea pharmacophore, Arch. Pharm. Res. 19 (1996) 570580.

18. S.H. Jung, S.J. Kwak, Planar structural requirement at 4-position of 1-arylsulfonyl-4-phenyl-4,5-dihydro2-imidazolones for their cytotoxicity, Arch. Pharm. Res. 20 (1997) 283-287.

19. S.H. Jung, H.S. Lee, J.S. Song, W.M. Kim, S.B. Han, C.W. Lee, M.S. Lee, D.R. Choi, J.A. Lee, Y.H. Chung, S.J. Yoon, E.Y. Moon, H.S. Hwang, S.K. Seong, D.K. Lee, Synthesis and antitumor activity of 4phenyl-1-arylsulfonyl imidazolidinones, Bioorg. Med. Chem. Lett. 8 (1998) 1547-1550. 
20. E.Y. Moon, S.K. Seong, S.H. Jung, M. Lee, D.K. Lee, D.K. Rhee, S. Pyo, S.J. Yoon, Antitumor activity of 4-phenyl-1-arylsulfonylimidazolidinone, DW2143, Cancer Lett. 140 (1999) 177-187.

21. E.Y. Moon, H.S. Hwang, C.H. Choi, S.H. Jung, S.J. Yoon, Effect of DW2282 on the induction of methemoglobinemia, hypoglycemia or WBC count and hematological changes, Arch. Phar. Res. 22 (1999) 565-570.

22. H.S. Hwang, E.Y. Moon, S.K. Seong, C.H. Choi, C.H. Chung, S.H. Jung, S.J. Yoon, Characterization of the anticancer activity of DW2282, a new anticancer agent, Anticancer. Res. 19 (1999) 5087-5093.

23. S.H. Jung, S.J. Kwak, N.D. Kim, S.U. Lee, C.O. Lee, Stereochemical requirement at 4-position of 4phenyl-1-arylsulfonylimidazolidinones for their cytotoxicities, Arch. Phar. Res. 23 (2000) 35-41.

24. S.H. Lee, K.L. Park, S.U. Choi, C.O. Lee, S.H. Jung, Effect of substituents on benzenesulfonyl motif of 4-phenyl-1-arylsulfonylimidazolidinones for their cytotoxicity, Arch. Pharm. Res. 23 (2000) 579-584.

25. I.W. Kim, S.H. Jung, Recognition of the importance of imidazolidinone motif for cytotoxicity of 4phenyl-1-arylsulfonylimidazolidinones using thiadiazolidine-1,1-dioxide analogs, Arch. Pharm. Res. 25 (2002) 421-427.

26. I. Kim, C. Lee, H. Kim, S. Jung, Importance of sulfonylimidazolidinone motif of 4-phenyl-1arylsulfonylimidazolidinones for their cytotoxicity: synthesis of 2-benzoyl-4-phenyl[1,2,5]thiazolidine1,1-dioxides and their cytotoxcity. Arch. Pharm. Res. 26 (2003) 9-14.

27. V. Zuliani, C. Carmi, M. Rivara, M. Fantini, A. Lodola, F. Vacondio, F. Bordi, P.V. Plazzi, A. Cavazzoni, M. Galetti, R.R. Alfieri, P.G. Petronini, M. Mor, 5-Benzylidene-hydantoins: synthesis and antiproliferative activity on A549 lung cancer cell line, Eur. J. Med. Chem. 44 (2009) 3471-3479.

28. D. Kaminskyy, B. Zimenkovsky, R. Lesyk, Synthesis and in vitro anticancer activity of 2,4azolidinedione-acetic acids derivatives, Eur. J. Med. Chem. 44 (2009) 3627-3636.

29. M. Mudit, M. Khanfar, A. Muralidharan, S. Thomas, G.V. Shah, R.W.M. van Soest, K.A. El Sayed, Discovery, design, and synthesis of anti-metastatic lead phenylmethylene hydantoins inspired by marine natural products, Bioorg. Med. Chem. 17 (2009) 1731-1738. 
30. C. Carmi, A. Cavazzoni, V. Zuliani, A. Lodola, F. Bordi, P.V. Plazzi, R.R. Alfieri, P.G. Petronini, M. Mor, 5-benzylidene-hydantoins as new EGFR inhibitors with antiproliferative activity, Bioorg. Med. Chem. Lett. 16 (2006) 4021-4025.

31. Z. Rajic, B. Zorc, S. Raic-Malic, K. Ester, M. Kralj, K. Pavelic, J. Balzarini, E. De Clercq, M. Mintas, Hydantoin derivatives of L- and D-amino acids: synthesis and evaluation of their antiviral and antitumoral activity, Molecules 11 (2006) 837-848.

32. P. Singh, M. Kaur, P. Verma, Design, synthesis and anticancer activities of hybrids of indole and barbituric acids--identification of highly promising leads, Bioorg. Med. Chem. Lett. 19 (2009) 3054-3058.

33. D.J. Guerin, D. Mazeas, M.S. Musale, N.F.M. Naguib, O.N. Al Safarjalani, M.H. el Kouni, R.P. Panzica, Uridine phosphorylase inhibitors: chemical modification of benzyloxybenzyl-barbituric acid and its effects on urdpase inhibition, Bioorg. Med. Chem. Lett. 9 (1999) 1477-1480, and references cited therein.

34. A.R. Shrestha, T. Shindo, N. Ashida, T. Nagamatsu, Synthesis, biological active molecular design, and molecular docking study of novel deazaflavin-cholestane hybrid compounds, Bioorg. Med. Chem. 16 (2008) 8685-8696.

35. H.-Y.P. Choo, M. Kim, S.K. Lee, S.W. Kim, S.W. Chung, Solid-phase combinatorial synthesis and cytotoxicity of 3-aryl-2,4-quinazolindiones, Bioorg Med. Chem. 10 (2002) 517-523.

36. Billek, G. Über die Kondensation aromatischer Aldehyde mit Hydantoin, Monatsh. Chem. 92 (1961) 352360.

37. J.C. Thenmozhiyal, P.T. Wong, W.K. Chui, Anticonvulsant activity of phenylmethylenehydantoins: a structure-activity relationship study, J. Med. Chem. 47 (2004) 1527-1535.

38. Rabjon, N., Organic Syntheses, Coll. Vol. IV, John Wiley and Sons Inc., Canada, 1963, p49.

39. Vogel, A. I., Vogel's Textbook of Practical Organic Chemistry, 5th ed., Longman, London, 1989, p963.

40. G. Brückmann, S.D. Isaacs, Preparation and properties of new derivatives of alloxan, J. Am. Chem. Soc. 71 (1949) 390-392.

41. M. Kurihara, N. Yoda, Molecular rearrangement in polyphosphoric acid I. Formation of 1, 2-dihydro-2phenylimino-4H-3,1-benzoxazin-4-one and rearrangement to 3-phenyl-2,4(1H,3H)-quinazolinedione in polyphosphoric acid. Tetrahedron Lett. 30 (1965) 2597-2606. 
42. S. Profeta, N.L. Allinger, Molecular mechanics calculations on aliphatic amines J. Am. Chem. Soc. 107 (1985) 1907-1918.

43. T.A. Halgren, Merck molecular force field. I. Basis, form, scope, parameterization, and performance of MMFF94, J. Comput. Chem. 17 (1996) 490-519.

44. MOE 2009.10 of Chemical Computing Group. Inc.

45. M.J.S. Dewar, E.G. Zoebisch, E.F. Healy, J.J.P. Stewart, Development and use of quantum mechanical molecular models. 76. AM1: a new general purpose quantum mechanical molecular model, J. Am. Chem. Soc. 107 (1985) 3902-3909.

46. A.I. Khodair, P.A. Bertrand, A new approach to the synthesis of substituted 4-imidazolidinones as potential antiviral and antitumor agents, Tetrahedron, 54 (1998) 4859-4872.

47. C.A. Lipinski, F. Lombardo, B.W. Dominy, P.J. Feeney, Experimental and computational approaches to estimate solubility and permeability in drug discovery and development settings, Adv. Drug Deliv. Rev. 46 (2001) 3-26.

48. D.E. Clark, S.D. Pickett, Computational methods for the prediction of 'drug-likeness', Drug Discov. Today 5 (2000) 49-58.

49. Pharma Algorithms (web edition), Toronto, Canada, 2009.

50. T.L. Moda, L.G. Torres, A.E. Carrara, A.D. Andricopulo. PK/DB: database for pharmacokinetic properties and predictive in silico ADME models, Bioinformatics 24 (2008) 2270-2271.

51. G. Wolber, T. Seidel, F. Bendix, T. Langer, Molecule-pharmacophore superpositioning and pattern matching in computational drug design, Drug Discov. Today 13 (2008) 23-29.

52. A.M. Al-Obaid, S.G. Abdel-Hamide, H.A. El-Kashef, A.A.-M. Abdel-Aziz, A.S. El-Azab, H.A. AlKhamees, H.I. El-Subbagh, Substituted quinazolines, part 3. Synthesis, in vitro antitumor activity and molecular modeling study of certain 2-thieno-4(3H)-quinazolinone analogs, Eur. J. Med. Chem. 44 (2009) 2379-2391.

53. H.-Y.P. Choo, J.-S. Lim, Y. Kam, S.Y. Kim, J. Lee, A comparative study of quantitative structure activity relationship methods based on antitumor diarylsulfonylureas, Eur. J. Med. Chem. 36 (2001) 829-836. 
54. H.-Y. P. Choo, S. Choi, S.-H. Jung, H.Y. Koh, A.N. Pae, The 3D-QSAR study of antitumor arylsulfonylimidazolidinone derivatives by CoMFA and CoMSIA, Bioorg. Med. Chem. 11 (2003) 45854589.

55. M.R. Grever, S.A. Sehepartz, B.A. Chabners, The National Cancer Institute: cancer drug discovery and development program, Semin. Oncol. 19 (1992) 622-638.

56. A. Monks, D. Schudiero, P. Skehan, R. Shoemaker, K. Paull, D. Vistica, C. Hose, J. Langley, P. Cronise, A. Vaigro-Wolff, M. Gray-Goodrich, H. Campbell, J. Mayo, M. Boyd, Feasibility of a high-flux anticancer drug screen using a diverse panel of cultured human tumor cell lines. J. Natl. Cancer Inst. 83 (1991) 757-766.

57. M.R. Boyd, K.D. Paull, Some practical considerations and applications of the National Cancer Institute in vitro anticancer drug discovery screen. Drug Dev. Res. 34 (1995) 91-109. 
Table 1. Physical properties, yields and molecular formulae of the synthesized compounds 3a-r.

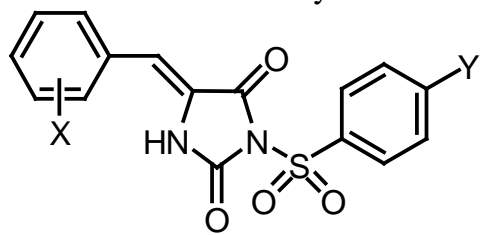

\begin{tabular}{|c|c|c|c|c|c|c|c|c|}
\hline \multirow{2}{*}{$\begin{array}{l}\text { Comp. } \\
\text { No. }\end{array}$} & \multirow[b]{2}{*}{$\mathbf{X}$} & \multirow[b]{2}{*}{$\mathbf{Y}$} & \multirow{2}{*}{$\begin{array}{c}\text { Yield } \\
\%\end{array}$} & \multirow{2}{*}{$\underset{\mathrm{C}}{\mathbf{M} . P .}$} & \multirow{2}{*}{$\begin{array}{l}\text { Mol. Formula } \\
\text { (Mol. Wt) }\end{array}$} & \multicolumn{3}{|c|}{ Analysis } \\
\hline & & & & & & Elm. & Calc. & Found \\
\hline 3a & $\mathrm{H}$ & $\mathrm{H}$ & 68 & $274-5$ & $\begin{array}{c}\mathrm{C}_{16} \mathrm{H}_{12} \mathrm{~N}_{2} \mathrm{O}_{4} \mathrm{~S} \\
(328.34)\end{array}$ & $\begin{array}{l}\mathrm{C} \\
\mathrm{H} \\
\mathrm{S}\end{array}$ & $\begin{array}{c}58.53 \\
3.68 \\
9.77\end{array}$ & $\begin{array}{c}58.72 \\
3.28 \\
9.91\end{array}$ \\
\hline $3 \mathbf{b}$ & $\mathrm{H}$ & $\mathrm{Cl}$ & 76 & $286-8$ & $\begin{array}{c}\mathrm{C}_{16} \mathrm{H}_{11} \mathrm{ClN}_{2} \mathrm{O}_{4} \mathrm{~S} \\
(362.79)\end{array}$ & $\begin{array}{l}\mathrm{C} \\
\mathrm{H} \\
\mathrm{N}\end{array}$ & $\begin{array}{l}52.97 \\
3.06 \\
7.72\end{array}$ & $\begin{array}{c}53.33 \\
3.26 \\
7.42\end{array}$ \\
\hline 3c & $\mathrm{H}$ & $\mathrm{CH}_{3}$ & 63 & $253-4$ & $\begin{array}{c}\mathrm{C}_{17} \mathrm{H}_{14} \mathrm{~N}_{2} \mathrm{O}_{4} \mathrm{~S} \\
\quad(342.37)\end{array}$ & $\begin{array}{l}\mathrm{C} \\
\mathrm{H} \\
\mathrm{N}\end{array}$ & $\begin{array}{c}59.64 \\
4.12 \\
8.18\end{array}$ & $\begin{array}{c}59.82 \\
4.06 \\
8.23\end{array}$ \\
\hline 3d & $4-\mathrm{Br}$ & $\mathrm{H}$ & 41 & $296-7$ & $\begin{array}{c}\mathrm{C}_{16} \mathrm{H}_{11} \mathrm{BrN}_{2} \mathrm{O}_{4} \mathrm{~S} \\
(407.24)\end{array}$ & $\begin{array}{l}\mathrm{C} \\
\mathrm{H} \\
\mathrm{S}\end{array}$ & $\begin{array}{l}47.19 \\
2.72 \\
7.87\end{array}$ & $\begin{array}{l}48.31 \\
2.55 \\
8.03\end{array}$ \\
\hline 3e & $4-\mathrm{Br}$ & $\mathrm{Cl}$ & 33 & $330-1$ & $\begin{array}{c}\mathrm{C}_{16} \mathrm{H}_{10} \mathrm{BrClN}_{2} \mathrm{O}_{4} \mathrm{~S} \\
(441.68)\end{array}$ & $\begin{array}{l}\mathrm{C} \\
\mathrm{H} \\
\mathrm{N}\end{array}$ & $\begin{array}{c}43.51 \\
2.28 \\
6.34\end{array}$ & $\begin{array}{l}44.02 \\
2.57 \\
6.11\end{array}$ \\
\hline $3 f$ & $4-\mathrm{Br}$ & $\mathrm{CH}_{3}$ & 37 & $294-5$ & $\begin{array}{c}\mathrm{C}_{17} \mathrm{H}_{13} \mathrm{BrN}_{2} \mathrm{O}_{4} \mathrm{~S} \\
(421.27)\end{array}$ & $\begin{array}{l}\mathrm{C} \\
\mathrm{H} \\
\mathrm{N}\end{array}$ & $\begin{array}{c}48.47 \\
3.11 \\
6.65\end{array}$ & $\begin{array}{l}49.14 \\
3.02 \\
6.79\end{array}$ \\
\hline $3 g$ & $4-\mathrm{Cl}$ & $\mathrm{H}$ & 38 & $300-2$ & $\begin{array}{c}\mathrm{C}_{16} \mathrm{H}_{11} \mathrm{ClN}_{2} \mathrm{O}_{4} \mathrm{~S} \\
(362.79)\end{array}$ & $\begin{array}{l}\mathrm{C} \\
\mathrm{H} \\
\mathrm{N}\end{array}$ & $\begin{array}{c}52.97 \\
3.06 \\
7.72\end{array}$ & $\begin{array}{c}54.17 \\
3.32 \\
7.48\end{array}$ \\
\hline $3 h$ & $4-\mathrm{Cl}$ & $\mathrm{Cl}$ & 41 & $326-9$ & $\begin{array}{c}\mathrm{C}_{16} \mathrm{H}_{10} \mathrm{Cl}_{2} \mathrm{~N}_{2} \mathrm{O}_{4} \mathrm{~S} \\
(397.23)\end{array}$ & $\begin{array}{l}\mathrm{C} \\
\mathrm{H} \\
\mathrm{N}\end{array}$ & $\begin{array}{c}48.38 \\
2.54 \\
7.05\end{array}$ & $\begin{array}{c}48.22 \\
2.64 \\
6.99\end{array}$ \\
\hline $3 \mathbf{i}$ & $4-\mathrm{Cl}$ & $\mathrm{CH}_{3}$ & 40 & $310-1$ & $\begin{array}{c}\mathrm{C}_{17} \mathrm{H}_{13} \mathrm{ClN}_{2} \mathrm{O}_{4} \mathrm{~S} \\
(376.81)\end{array}$ & $\begin{array}{l}\mathrm{C} \\
\mathrm{H} \\
\mathrm{S}\end{array}$ & $\begin{array}{c}54.19 \\
3.48 \\
8.51\end{array}$ & $\begin{array}{c}53.74 \\
3.38 \\
8.74\end{array}$ \\
\hline $3 \mathbf{j}$ & $3-\mathrm{NO}_{2}$ & $\mathrm{H}$ & 52 & $277-9$ & $\begin{array}{c}\mathrm{C}_{16} \mathrm{H}_{11} \mathrm{~N}_{3} \mathrm{O}_{6} \mathrm{~S} \\
(373.34)\end{array}$ & $\begin{array}{l}\mathrm{C} \\
\mathrm{H} \\
\mathrm{S}\end{array}$ & $\begin{array}{c}51.47 \\
2.97 \\
8.59\end{array}$ & $\begin{array}{c}51.68 \\
2.72 \\
8.71\end{array}$ \\
\hline $3 \mathbf{k}$ & $3-\mathrm{NO}_{2}$ & $\mathrm{Cl}$ & 48 & $300-2$ & $\begin{array}{c}\mathrm{C}_{16} \mathrm{H}_{10} \mathrm{ClN}_{3} \mathrm{O}_{6} \mathrm{~S} \\
(407.79)\end{array}$ & $\begin{array}{l}\mathrm{C} \\
\mathrm{H} \\
\mathrm{N}\end{array}$ & $\begin{array}{l}47.13 \\
2.47 \\
10.3\end{array}$ & $\begin{array}{c}46.88 \\
2.61 \\
10.47\end{array}$ \\
\hline $3 \mathbf{l}$ & $3-\mathrm{NO}_{2}$ & $\mathrm{CH}_{3}$ & 45 & $291-3$ & $\begin{array}{c}\mathrm{C}_{17} \mathrm{H}_{13} \mathrm{~N}_{3} \mathrm{O}_{6} \mathrm{~S} \\
(387.37)\end{array}$ & $\begin{array}{l}\mathrm{C} \\
\mathrm{H} \\
\mathrm{N}\end{array}$ & $\begin{array}{c}52.71 \\
3.38 \\
10.85\end{array}$ & $\begin{array}{c}52.44 \\
3.61 \\
10.93\end{array}$ \\
\hline $3 \mathrm{~m}$ & $4-\mathrm{OCH}_{3}$ & $\mathrm{H}$ & 65 & $270-2$ & $\begin{array}{c}\mathrm{C}_{17} \mathrm{H}_{14} \mathrm{~N}_{2} \mathrm{O}_{5} \mathrm{~S} \\
(358.37)\end{array}$ & $\begin{array}{l}\mathrm{C} \\
\mathrm{H} \\
\mathrm{N}\end{array}$ & $\begin{array}{c}56.98 \\
3.94 \\
7.82\end{array}$ & $\begin{array}{l}56.49 \\
3.70 \\
7.66\end{array}$ \\
\hline $3 n$ & $4-\mathrm{OCH}_{3}$ & $\mathrm{Cl}$ & 71 & $290-2$ & $\begin{array}{c}\mathrm{C}_{17} \mathrm{H}_{13} \mathrm{ClN}_{2} \mathrm{O}_{5} \mathrm{~S} \\
(392.81)\end{array}$ & $\begin{array}{l}\mathrm{C} \\
\mathrm{H} \\
\mathrm{S}\end{array}$ & $\begin{array}{c}51.98 \\
3.34 \\
8.16\end{array}$ & $\begin{array}{c}51.66 \\
3.19 \\
8.41\end{array}$ \\
\hline 30 & $4-\mathrm{OCH}_{3}$ & $\mathrm{CH}_{3}$ & 62 & $255-6$ & $\begin{array}{c}\mathrm{C}_{18} \mathrm{H}_{16} \mathrm{~N}_{2} \mathrm{O}_{5} \mathrm{~S} \\
(372.40)\end{array}$ & $\begin{array}{l}\mathrm{C} \\
\mathrm{H} \\
\mathrm{S}\end{array}$ & $\begin{array}{c}58.05 \\
4.33 \\
8.61\end{array}$ & $\begin{array}{l}57.78 \\
4.50 \\
8.54\end{array}$ \\
\hline $3 p$ & $4-\left(\mathrm{CH}_{3}\right)_{2} \mathrm{~N}$ & $\mathrm{H}$ & 22 & $275-7$ & $\begin{array}{c}\mathrm{C}_{18} \mathrm{H}_{17} \mathrm{~N}_{3} \mathrm{O}_{4} \mathrm{~S} \\
(371.41)\end{array}$ & $\begin{array}{l}\mathrm{C} \\
\mathrm{H} \\
\mathrm{N}\end{array}$ & $\begin{array}{c}58.21 \\
4.61 \\
11.31\end{array}$ & $\begin{array}{c}58.61 \\
4.87 \\
11.06\end{array}$ \\
\hline $3 q$ & $4-\left(\mathrm{CH}_{3}\right)_{2} \mathrm{~N}$ & $\mathrm{Cl}$ & 18 & $262-4$ & $\begin{array}{c}\mathrm{C}_{18} \mathrm{H}_{16} \mathrm{ClN}_{3} \mathrm{O}_{4} \mathrm{~S} \\
(405.86)\end{array}$ & $\begin{array}{l}\mathrm{C} \\
\mathrm{H} \\
\mathrm{N}\end{array}$ & $\begin{array}{c}53.27 \\
3.97 \\
10.35\end{array}$ & $\begin{array}{c}53.88 \\
3.52 \\
10.60\end{array}$ \\
\hline $3 r$ & $4-\left(\mathrm{CH}_{3}\right)_{2} \mathrm{~N}$ & $\mathrm{CH}_{3}$ & 18 & $292-3$ & $\begin{array}{c}\mathrm{C}_{19} \mathrm{H}_{19} \mathrm{~N}_{3} \mathrm{O}_{4} \mathrm{~S} \\
(385.44)\end{array}$ & $\begin{array}{l}\mathrm{C} \\
\mathrm{H} \\
\mathrm{S} \\
\end{array}$ & $\begin{array}{c}59.21 \\
4.97 \\
8.32 \\
\end{array}$ & $\begin{array}{c}59.09 \\
4.68 \\
8.61 \\
\end{array}$ \\
\hline
\end{tabular}


Table 2. Physical properties, yields and molecular formulae of the synthesized compounds 6a-l.<smiles>[X]c1ccc(N2C(=O)CC(=O)N(S(=O)(=O)c3ccc([X])cc3)C2=O)cc1</smiles>

\begin{tabular}{|c|c|c|c|c|c|c|c|c|}
\hline \multirow{2}{*}{$\begin{array}{l}\text { Comp. } \\
\text { No. }\end{array}$} & \multirow[b]{2}{*}{$\mathbf{X}$} & \multirow[b]{2}{*}{$\mathbf{Y}$} & \multirow{2}{*}{$\begin{array}{c}\text { Yield } \\
\%\end{array}$} & \multirow{2}{*}{$\underset{{ }^{\circ} \mathrm{C}}{\mathrm{M} . P .}$} & \multirow{2}{*}{$\begin{array}{l}\text { Mol. Formula } \\
\text { (Mol. Wt) }\end{array}$} & \multicolumn{3}{|c|}{ Analysis } \\
\hline & & & & & & Elm. & Calc. & Found \\
\hline 6a & $\mathrm{H}$ & $\mathrm{H}$ & 59 & $255-7$ & $\begin{array}{c}\mathrm{C}_{16} \mathrm{H}_{12} \mathrm{~N}_{2} \mathrm{O}_{5} \mathrm{~S} \\
(344.34)\end{array}$ & $\begin{array}{l}\mathrm{C} \\
\mathrm{H} \\
\mathrm{N}\end{array}$ & $\begin{array}{c}55.81 \\
3.51 \\
8.14\end{array}$ & $\begin{array}{c}55.61 \\
3.82 \\
8.36\end{array}$ \\
\hline 6b & $\mathrm{H}$ & $\mathrm{Cl}$ & 51 & $218-20$ & $\begin{array}{c}\mathrm{C}_{16} \mathrm{H}_{11} \mathrm{ClN}_{2} \mathrm{O}_{5} \mathrm{~S} \\
(378.79)\end{array}$ & $\begin{array}{l}\mathrm{C} \\
\mathrm{H} \\
\mathrm{S}\end{array}$ & $\begin{array}{c}50.73 \\
2.93 \\
8.47\end{array}$ & $\begin{array}{c}50.41 \\
2.68 \\
8.90\end{array}$ \\
\hline 6c & $\mathrm{H}$ & $\mathrm{CH}_{3}$ & 54 & $248-50$ & $\begin{array}{c}\mathrm{C}_{17} \mathrm{H}_{14} \mathrm{~N}_{2} \mathrm{O}_{5} \mathrm{~S} \\
(358.37)\end{array}$ & $\begin{array}{l}\mathrm{C} \\
\mathrm{H} \\
\mathrm{N}\end{array}$ & $\begin{array}{c}56.98 \\
3.94 \\
7.82\end{array}$ & $\begin{array}{c}57.22 \\
3.70 \\
7.96\end{array}$ \\
\hline 6d & $\mathrm{Cl}$ & $\mathrm{H}$ & 47 & $230-2$ & $\begin{array}{c}\mathrm{C}_{16} \mathrm{H}_{11} \mathrm{ClN}_{2} \mathrm{O}_{5} \mathrm{~S} \\
(378.79)\end{array}$ & $\begin{array}{l}\mathrm{C} \\
\mathrm{H} \\
\mathrm{N}\end{array}$ & $\begin{array}{c}50.73 \\
2.93 \\
7.40\end{array}$ & $\begin{array}{c}50.42 \\
3.06 \\
7.28\end{array}$ \\
\hline $6 e$ & $\mathrm{Cl}$ & $\mathrm{Cl}$ & 44 & $232-4$ & $\begin{array}{c}\mathrm{C}_{16} \mathrm{H}_{10} \mathrm{C}_{12} \mathrm{~N}_{2} \mathrm{O}_{5} \mathrm{~S} \\
(413.23)\end{array}$ & $\begin{array}{l}\mathrm{C} \\
\mathrm{H} \\
\mathrm{S}\end{array}$ & $\begin{array}{c}46.50 \\
2.44 \\
7.76\end{array}$ & $\begin{array}{c}46.33 \\
2.61 \\
7.60\end{array}$ \\
\hline $6 f$ & $\mathrm{Cl}$ & $\mathrm{CH}_{3}$ & 42 & 243-5 & $\begin{array}{c}\mathrm{C}_{17} \mathrm{H}_{13} \mathrm{ClN}_{2} \mathrm{O}_{5} \mathrm{~S} \\
(392.81)\end{array}$ & $\begin{array}{l}\mathrm{C} \\
\mathrm{H} \\
\mathrm{S}\end{array}$ & $\begin{array}{c}51.98 \\
3.34 \\
8.16\end{array}$ & $\begin{array}{c}52.26 \\
3.39 \\
7.97\end{array}$ \\
\hline $6 g$ & $\mathrm{CH}_{3}$ & $\mathrm{H}$ & 33 & $242-3$ & $\begin{array}{c}\mathrm{C}_{17} \mathrm{H}_{14} \mathrm{~N}_{2} \mathrm{O}_{5} \mathrm{~S} \\
(358.37)\end{array}$ & $\begin{array}{l}\mathrm{C} \\
\mathrm{H} \\
\mathrm{S}\end{array}$ & $\begin{array}{l}56.98 \\
3.94 \\
8.95\end{array}$ & $\begin{array}{c}56.62 \\
3.75 \\
9.16\end{array}$ \\
\hline $6 h$ & $\mathrm{CH}_{3}$ & $\mathrm{Cl}$ & 38 & $247-8$ & $\begin{array}{c}\mathrm{C}_{17} \mathrm{H}_{13} \mathrm{ClN}_{2} \mathrm{O}_{5} \mathrm{~S} \\
(392.81)\end{array}$ & $\begin{array}{l}\mathrm{C} \\
\mathrm{H} \\
\mathrm{N}\end{array}$ & $\begin{array}{l}51.98 \\
3.34 \\
7.13\end{array}$ & $\begin{array}{c}51.62 \\
3.59 \\
7.33\end{array}$ \\
\hline $6 i$ & $\mathrm{CH}_{3}$ & $\mathrm{CH}_{3}$ & 39 & $252-4$ & $\begin{array}{c}\mathrm{C}_{18} \mathrm{H}_{16} \mathrm{~N}_{2} \mathrm{O}_{5} \mathrm{~S} \\
(372.40)\end{array}$ & $\begin{array}{l}\mathrm{C} \\
\mathrm{H} \\
\mathrm{N}\end{array}$ & $\begin{array}{c}58.05 \\
4.33 \\
7.52\end{array}$ & $\begin{array}{c}58.33 \\
4.09 \\
7.68\end{array}$ \\
\hline $6 j$ & $\mathrm{OCH}_{3}$ & $\mathrm{H}$ & 52 & $228-30$ & $\begin{array}{c}\mathrm{C}_{17} \mathrm{H}_{14} \mathrm{~N}_{2} \mathrm{O}_{6} \mathrm{~S} \\
(374.37)\end{array}$ & $\begin{array}{l}\mathrm{C} \\
\mathrm{H} \\
\mathrm{N}\end{array}$ & $\begin{array}{c}54.54 \\
3.77 \\
7.48\end{array}$ & $\begin{array}{c}54.23 \\
3.91 \\
7.52\end{array}$ \\
\hline $6 k$ & $\mathrm{OCH}_{3}$ & $\mathrm{Cl}$ & 46 & 246-8 & $\begin{array}{c}\mathrm{C}_{17} \mathrm{H}_{13} \mathrm{ClN}_{2} \mathrm{O}_{6} \mathrm{~S} \\
\text { (408.81) }\end{array}$ & $\begin{array}{l}\mathrm{C} \\
\mathrm{H} \\
\mathrm{S}\end{array}$ & $\begin{array}{c}49.95 \\
3.21 \\
7.84\end{array}$ & $\begin{array}{c}50.16 \\
3.42 \\
7.62\end{array}$ \\
\hline 61 & $\mathrm{OCH}_{3}$ & $\mathrm{CH}_{3}$ & 50 & $224-5$ & $\begin{array}{c}\mathrm{C}_{18} \mathrm{H}_{16} \mathrm{~N}_{2} \mathrm{O}_{6} \mathrm{~S} \\
(388.39)\end{array}$ & $\begin{array}{l}\mathrm{C} \\
\mathrm{H} \\
\mathrm{N}\end{array}$ & $\begin{array}{c}55.66 \\
4.15 \\
7.21\end{array}$ & $\begin{array}{c}55.41 \\
4.31 \\
7.06\end{array}$ \\
\hline
\end{tabular}


Table 3. Physical properties, yields and molecular formulae of the synthesized compounds 8a-l.

\begin{tabular}{|c|c|c|c|c|c|c|c|c|}
\hline \multirow{2}{*}{$\begin{array}{l}\text { Comp. } \\
\text { No. }\end{array}$} & \multirow[b]{2}{*}{$\mathbf{X}$} & \multirow[b]{2}{*}{$\mathbf{Y}$} & \multirow{2}{*}{$\begin{array}{c}\text { Yield } \\
\%\end{array}$} & \multirow{2}{*}{$\underset{{ }^{\circ} \mathbf{C}}{\mathbf{M . P}}$} & \multirow{2}{*}{$\begin{array}{l}\text { Mol. Formula } \\
\text { (Mol. Wt) }\end{array}$} & \multicolumn{3}{|c|}{ Analysis } \\
\hline & & & & & & Elm. & Calc. & Found \\
\hline $8 a$ & $\mathrm{H}$ & $\mathrm{H}$ & 49 & $195-6$ & $\begin{array}{c}\mathrm{C}_{20} \mathrm{H}_{14} \mathrm{~N}_{2} \mathrm{O}_{4} \mathrm{~S} \\
(378.40)\end{array}$ & $\begin{array}{l}\mathrm{C} \\
\mathrm{H} \\
\mathrm{N}\end{array}$ & $\begin{array}{c}63.48 \\
3.73 \\
7.40\end{array}$ & $\begin{array}{c}63.62 \\
3.51 \\
7.64\end{array}$ \\
\hline $\mathbf{8 b}$ & $\mathrm{H}$ & $\mathrm{Cl}$ & 27 & $220-2$ & $\begin{array}{c}\mathrm{C}_{20} \mathrm{H}_{13} \mathrm{ClN}_{2} \mathrm{O}_{4} \mathrm{~S} \\
(412.85)\end{array}$ & $\begin{array}{l}\mathrm{C} \\
\mathrm{H} \\
\mathrm{S}\end{array}$ & $\begin{array}{l}58.18 \\
3.17 \\
7.77\end{array}$ & $\begin{array}{l}58.26 \\
3.28 \\
7.42\end{array}$ \\
\hline $8 c$ & $\mathrm{H}$ & $\mathrm{CH}_{3}$ & 47 & $170-1$ & $\begin{array}{c}\mathrm{C}_{21} \mathrm{H}_{16} \mathrm{~N}_{2} \mathrm{O}_{4} \mathrm{~S} \\
(392.43)\end{array}$ & $\begin{array}{l}\mathrm{C} \\
\mathrm{H} \\
\mathrm{N}\end{array}$ & $\begin{array}{c}64.27 \\
4.11 \\
7.14\end{array}$ & $\begin{array}{c}64.39 \\
4.33 \\
7.01\end{array}$ \\
\hline $8 d$ & $\mathrm{Cl}$ & $\mathrm{H}$ & 26 & $180-1$ & $\begin{array}{c}\mathrm{C}_{20} \mathrm{H}_{13} \mathrm{ClN}_{2} \mathrm{O}_{4} \mathrm{~S} \\
(412.85)\end{array}$ & $\begin{array}{l}\mathrm{C} \\
\mathrm{H} \\
\mathrm{N}\end{array}$ & $\begin{array}{l}58.18 \\
3.17 \\
6.79\end{array}$ & $\begin{array}{l}57.82 \\
3.35 \\
6.64\end{array}$ \\
\hline $8 e$ & $\mathrm{Cl}$ & $\mathrm{Cl}$ & 19 & $213-5$ & $\begin{array}{c}\mathrm{C}_{20} \mathrm{H}_{12} \mathrm{Cl}_{2} \mathrm{~N}_{2} \mathrm{O}_{4} \mathrm{~S} \\
(447.29)\end{array}$ & $\begin{array}{l}\mathrm{C} \\
\mathrm{H} \\
\mathrm{N}\end{array}$ & $\begin{array}{l}53.70 \\
2.70 \\
6.26\end{array}$ & $\begin{array}{c}53.89 \\
2.45 \\
6.29\end{array}$ \\
\hline $8 f$ & $\mathrm{Cl}$ & $\mathrm{CH}_{3}$ & 24 & $192-3$ & $\begin{array}{c}\mathrm{C}_{21} \mathrm{H}_{15} \mathrm{ClN}_{2} \mathrm{O}_{4} \mathrm{~S} \\
(426.87)\end{array}$ & $\begin{array}{l}\mathrm{C} \\
\mathrm{H} \\
\mathrm{S}\end{array}$ & $\begin{array}{l}59.09 \\
3.54 \\
7.51\end{array}$ & $\begin{array}{l}59.11 \\
3.41 \\
7.39\end{array}$ \\
\hline $8 g$ & $\mathrm{CH}_{3}$ & $\mathrm{H}$ & 29 & $194-6$ & $\begin{array}{c}\mathrm{C}_{21} \mathrm{H}_{16} \mathrm{~N}_{2} \mathrm{O}_{4} \mathrm{~S} \\
(392.43)\end{array}$ & $\begin{array}{l}\mathrm{C} \\
\mathrm{H} \\
\mathrm{S}\end{array}$ & $\begin{array}{c}64.27 \\
4.11 \\
8.17\end{array}$ & $\begin{array}{l}64.02 \\
4.37 \\
7.86\end{array}$ \\
\hline $\mathbf{8 h}$ & $\mathrm{CH}_{3}$ & $\mathrm{Cl}$ & 22 & $202-4$ & $\begin{array}{c}\mathrm{C}_{21} \mathrm{H}_{15} \mathrm{ClN}_{2} \mathrm{O}_{4} \mathrm{~S} \\
(426.87)\end{array}$ & $\begin{array}{l}\mathrm{C} \\
\mathrm{H} \\
\mathrm{N}\end{array}$ & $\begin{array}{c}59.09 \\
3.54 \\
6.56\end{array}$ & $\begin{array}{l}59.31 \\
3.62 \\
6.44\end{array}$ \\
\hline $8 \mathbf{i}$ & $\mathrm{CH}_{3}$ & $\mathrm{CH}_{3}$ & 33 & $216-7$ & $\begin{array}{c}\mathrm{C}_{22} \mathrm{H}_{18} \mathrm{~N}_{2} \mathrm{O}_{4} \mathrm{~S} \\
(406.45)\end{array}$ & $\begin{array}{l}\mathrm{C} \\
\mathrm{H} \\
\mathrm{N}\end{array}$ & $\begin{array}{c}65.01 \\
4.46 \\
6.89\end{array}$ & $\begin{array}{l}65.26 \\
4.29 \\
6.92\end{array}$ \\
\hline $8 \mathbf{j}$ & $\mathrm{OCH}_{3}$ & $\mathrm{H}$ & 32 & $190-2$ & $\begin{array}{l}\mathrm{C}_{21} \mathrm{H}_{16} \mathrm{~N}_{2} \mathrm{O}_{5} \mathrm{~S} \\
\quad(408.43)\end{array}$ & $\begin{array}{l}\mathrm{C} \\
\mathrm{H} \\
\mathrm{N}\end{array}$ & $\begin{array}{l}61.76 \\
3.95 \\
6.86\end{array}$ & $\begin{array}{l}62.03 \\
3.77 \\
6.92\end{array}$ \\
\hline $\mathbf{8 k}$ & $\mathrm{OCH}_{3}$ & $\mathrm{Cl}$ & 21 & $167-8$ & $\begin{array}{c}\mathrm{C}_{21} \mathrm{H}_{15} \mathrm{ClN}_{2} \mathrm{O}_{5} \mathrm{~S} \\
(442.87)\end{array}$ & $\begin{array}{l}\mathrm{C} \\
\mathrm{H} \\
\mathrm{S}\end{array}$ & $\begin{array}{l}56.95 \\
3.41 \\
7.24\end{array}$ & $\begin{array}{l}56.66 \\
3.62 \\
7.52\end{array}$ \\
\hline 81 & $\mathrm{OCH}_{3}$ & $\mathrm{CH}_{3}$ & 30 & $178-9$ & $\begin{array}{l}\mathrm{C}_{22} \mathrm{H}_{18} \mathrm{~N}_{2} \mathrm{O}_{5} \mathrm{~S} \\
\quad(422.45)\end{array}$ & $\begin{array}{l}\mathrm{C} \\
\mathrm{H} \\
\mathrm{S}\end{array}$ & $\begin{array}{l}62.55 \\
4.29 \\
7.59\end{array}$ & $\begin{array}{c}62.21 \\
4.44 \\
7.34\end{array}$ \\
\hline
\end{tabular}


Table 4. The percentages of growth inhibition of the thirteen selected compounds over the most sensitive tumor cell lines.

\begin{tabular}{cccccc}
\hline $\begin{array}{c}\text { Comp. } \\
\text { No }\end{array}$ & $\begin{array}{c}\text { IGROV1 } \\
(\mathbf{\% )}\end{array}$ & $\begin{array}{c}\text { OVCAR-8 } \\
(\mathbf{\%})\end{array}$ & $\begin{array}{c}\text { RXF393 } \\
\mathbf{( \% )}\end{array}$ & $\begin{array}{c}\text { M14 } \\
(\mathbf{\%})\end{array}$ & $\begin{array}{c}\text { SK-MEL-2 } \\
(\mathbf{\%})\end{array}$ \\
\hline $\mathbf{3 b}$ & -9.05 & 3.88 & 0.92 & - & 11.71 \\
$\mathbf{3 g}$ & 1.13 & 6.79 & 7.42 & - & 19.95 \\
$\mathbf{3 h}$ & 80.99 & 4.01 & 76.2 & -20.14 & 17.63 \\
$\mathbf{3 m}$ & 114.67 & 5.23 & 96.49 & - & 11.57 \\
$\mathbf{3 n}$ & -1.97 & -1.23 & 84.12 & - & 8.98 \\
$\mathbf{3 p}$ & 106.89 & 1.17 & 80.28 & -2.88 & 3.98 \\
$\mathbf{3 q}$ & 97.89 & -8.63 & 95.74 & 199.62 & 1.29 \\
$\mathbf{6 b}$ & 34.14 & 58.86 & - & 8.19 & 65.1 \\
$\mathbf{6 f}$ & 65.6 & 54.13 & - & -7.35 & 73.93 \\
$\mathbf{6 k}$ & 76.55 & 42.41 & - & -3.04 & 61.07 \\
$\mathbf{8 a}$ & 86.78 & 33.24 & - & 1.02 & 70.4 \\
$\mathbf{8 b}$ & 91.42 & 40.85 & - & 17.36 & 79.27 \\
$\mathbf{8 j}$ & 63.16 & 26.02 & 42.95 & 56.53 & -43.25 \\
\hline
\end{tabular}

*The showed inhibition percentages are measured at a single concentration of $10 \mu \mathrm{M}$. \% inhibition is calculated by simple abstraction of the \% activity from $100.100 \%$ activity is the activity of cells in presence of test solvent only (DMSO). \% Inhibition between 100-200\% means that the compound has a lethal effect at cancer cells. 
Table 5. The percentages of growth inhibition of compound 3q over the full panel of tumor cell lines*

\begin{tabular}{|c|c|c|c|c|c|}
\hline $\begin{array}{c}\text { Cell line } \\
\text { type }\end{array}$ & Cell line name & $\begin{array}{c}\text { Inhibition } \\
\text { (\%) }\end{array}$ & $\begin{array}{c}\text { Cell line } \\
\text { type }\end{array}$ & Cell line name & $\begin{array}{c}\text { Inhibition } \\
(\%)\end{array}$ \\
\hline \multirow{7}{*}{ 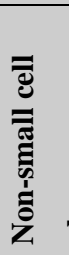 } & A549/ATCC & -8.84 & \multirow{5}{*}{ 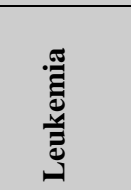 } & CCRF-CEM & 2.95 \\
\hline & EKVX & 14.7 & & HL-60(TB) & 4.85 \\
\hline & HOP-92 & 11.19 & & $\mathrm{~K}-562$ & 8.89 \\
\hline & NCI-H226 & -9.94 & & MOLT-4 & 11.54 \\
\hline & NCI-H23 & -1.8 & & RPMI-8226 & -6.72 \\
\hline & NCI-H322M & -16.53 & \multirow{9}{*}{ 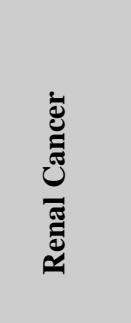 } & SR & 34.66 \\
\hline & NCI-H460 & -15.77 & & $786-0$ & -0.44 \\
\hline \multirow{7}{*}{ 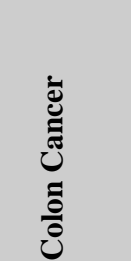 } & NCI-H522 & 9.78 & & A498 & -8.94 \\
\hline & COLO 205 & -14.66 & & $\mathrm{ACHN}$ & -5.38 \\
\hline & HCC-2998 & -54.05 & & CAKI-1 & -5.71 \\
\hline & HCT-116 & -3.55 & & RXF393 & 95.74 \\
\hline & HCT-15 & 1.55 & & SN12C & 0.04 \\
\hline & НT29 & -2.64 & & TK-10 & -33.42 \\
\hline & KM12 & -11.59 & & UO-31 & 5.5 \\
\hline \multirow{7}{*}{ 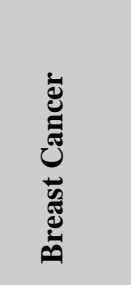 } & SW-620 & -1.96 & \multirow{8}{*}{ 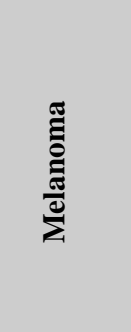 } & LOX IMVI & 6.44 \\
\hline & ВТ-549 & 4.63 & & M14 & 199.62 \\
\hline & HS $578 \mathrm{~T}$ & 14.08 & & MALME-3M & -49.66 \\
\hline & MCF7 & -6.37 & & SK-MEL-2 & 1.29 \\
\hline & $\begin{array}{l}\text { MDA-MB- } \\
\text { 231/ATCC }\end{array}$ & -2.18 & & SK-MEL-28 & -4.69 \\
\hline & MDA-MB-435 & -4.89 & & SK-MEL-5 & -3.11 \\
\hline & NCI/ADR-RES & -6.33 & & UACC-257 & -1.01 \\
\hline \multirow{7}{*}{ 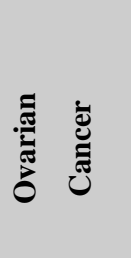 } & T-47D & -9.29 & & UACC-62 & 3.93 \\
\hline & IGROV1 & 97.89 & \multirow{6}{*}{ 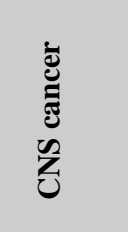 } & SF-268 & -21.61 \\
\hline & OVCAR-3 & -13.88 & & SF-295 & -8.92 \\
\hline & OVCAR-4 & 7.93 & & SF-539 & -0.9 \\
\hline & OVCAR-5 & -4.44 & & SNB-19 & -6.78 \\
\hline & OVCAR-8 & -8.63 & & SNB-75 & -5.55 \\
\hline & SK-OV-3 & -8.45 & & U251 & -0.69 \\
\hline Prostate & DU-145 & -4.25 & & & \\
\hline Cancer & PC-3 & -6.65 & & & \\
\hline
\end{tabular}

${ }^{*}$ The showed inhibition percentages are measured at a single concentration of $10 \mu \mathrm{M} . \%$ inhibition is calculated by simple abstraction of the \% activity from $100.100 \%$ activity is the activity of cells in presence of test solvent only (DMSO). \% Inhibition between 100-200\% means that the compound has a lethal effect at cancer cells. 
Table 6. Molar refractometry and calculated Lipinski’s rule of five for the tested compounds.

\begin{tabular}{|c|c|c|c|c|c|c|c|c|}
\hline \multirow{2}{*}{$\begin{array}{c}\text { Comp } \\
\text { No }\end{array}$} & \multirow{2}{*}{$\begin{array}{c}\text { IGROV1 }^{\mathbf{a}} \\
\text { (\% Inhibition) }\end{array}$} & \multirow{2}{*}{$\mathbf{M R}^{\mathbf{b}}$} & \multicolumn{5}{|c|}{ Parameter } & \multirow{2}{*}{$N_{\text {violatios }}{ }^{\mathrm{h}}$} \\
\hline & & & $\log P^{c}$ & TPSA $^{\mathrm{d}}$ & $\mathbf{M W}^{\mathrm{e}}$ & $\boldsymbol{n O N ^ { f }}$ & $n \mathrm{OHNH}^{\mathrm{g}}$ & \\
\hline $3 \mathbf{b}$ & -9.05 & 88.7 & 2.5 & 83.6 & 362.8 & 6 & 1 & 0 \\
\hline $3 g$ & 1.13 & 88.7 & 2.5 & 83.6 & 362.8 & 6 & 1 & 0 \\
\hline $3 \mathbf{h}$ & 80.99 & 93.3 & 3.3 & 83.6 & 397.2 & 6 & 1 & 0 \\
\hline $3 m$ & 114.67 & 91.4 & 1.8 & 92.8 & 358.4 & 7 & 1 & 0 \\
\hline $3 n$ & -1.97 & 96.0 & 2.5 & 92.8 & 392.8 & 7 & 1 & 0 \\
\hline $3 p$ & 106.89 & 99.3 & 2.0 & 86.8 & 371.4 & 7 & 1 & 0 \\
\hline $3 q$ & 97.89 & 103.9 & 2.7 & 86.8 & 405.9 & 7 & 1 & 0 \\
\hline $6 b$ & 34.14 & 86.4 & 2.8 & 91.8 & 378.8 & 7 & 0 & 0 \\
\hline $6 f$ & 65.6 & 92.3 & 3.3 & 91.8 & 392.8 & 7 & 0 & 0 \\
\hline $6 k$ & 76.55 & 93.7 & 2.7 & 101.1 & 408.8 & 8 & 0 & 0 \\
\hline $8 a$ & 86.78 & 98.1 & 3.8 & 74.8 & 378.4 & 6 & 0 & 0 \\
\hline $8 \mathbf{b}$ & 91.42 & 102.7 & 4.5 & 74.8 & 412.9 & 6 & 0 & 0 \\
\hline $8 \mathbf{j}$ & 63.16 & 105.4 & 3.7 & 84.0 & 408.4 & 7 & 0 & 0 \\
\hline
\end{tabular}

${ }^{\mathrm{a}}$ Data taken from table 1, ${ }^{\mathrm{b}}$ Molar refractometry, 'Calculated lipophilicity, ${ }^{\mathrm{d}}$ Total polar surface area, ${ }^{\mathrm{e}}$ Molecular weigh, ${ }^{\mathrm{f}}$ Number of hydrogen bond acceptor, ${ }^{\mathrm{g}}$ Number of hydrogen bond donor, ${ }^{\mathrm{h}}$ Number of violation from Lipinski's rule of five. 
Table 7. The predicted ADME-Tox of the proposed and reported antitumor agents.

\begin{tabular}{|c|c|c|c|c|c|c|c|c|c|c|c|c|c|}
\hline ADME-Tox ${ }^{3}$ & $3 \mathbf{h}$ & $3 m$ & $3 p$ & $3 q$ & $6 b$ & $6 f$ & $6 \mathbf{k}$ & $8 a$ & $8 b$ & $8 \mathbf{j}$ & $\mathbf{A}$ & D & $\mathbf{E}$ \\
\hline Solubility (LogS) & $\begin{array}{l}-6.11 \\
( \pm 0.5)\end{array}$ & $\begin{array}{l}-4.76 \\
( \pm 0.5)\end{array}$ & $\begin{array}{l}-4.96 \\
( \pm 0.5)\end{array}$ & $\begin{array}{l}-5.83 \\
( \pm 0.5)\end{array}$ & $\begin{array}{l}-4.48 \\
( \pm 0.5)\end{array}$ & $\begin{array}{l}-4.92 \\
( \pm 0.5)\end{array}$ & $\begin{array}{l}-4.87 \\
( \pm 0.5)\end{array}$ & $\begin{array}{l}-5.56 \\
( \pm 0.5)\end{array}$ & $\begin{array}{l}-6.44 \\
( \pm 0.5)\end{array}$ & $\begin{array}{l}-5.95 \\
( \pm 0.5)\end{array}$ & $\begin{array}{l}-5.47 \\
( \pm 0.5)\end{array}$ & $\begin{array}{l}-4.99 \\
( \pm 0.5)\end{array}$ & $\begin{array}{l}-6.28 \\
( \pm 0.5)\end{array}$ \\
\hline$F(\%)^{\mathrm{a}}$ & $97.38( \pm 15)$ & $98.27( \pm 15)$ & $\begin{array}{l}83.78 \\
( \pm 15)\end{array}$ & $\begin{array}{l}84.27 \\
( \pm 15)\end{array}$ & $\begin{array}{c}107.62 \\
( \pm 15)\end{array}$ & $\begin{array}{l}107.25 \\
( \pm 15)\end{array}$ & $\begin{array}{c}110.04 \\
( \pm 15)\end{array}$ & $56.88( \pm 15)$ & $\begin{array}{l}56.18 \\
( \pm 15)\end{array}$ & $\begin{array}{l}57.08 \\
( \pm 15)\end{array}$ & $81.41( \pm 15)$ & $69.04( \pm 15)$ & $51.15( \pm 15)$ \\
\hline HIA (\%) & $\begin{array}{l}95.17 \\
( \pm 13)\end{array}$ & $\begin{array}{l}95.17 \\
( \pm 13)\end{array}$ & $\begin{array}{l}99.02 \\
( \pm 13)\end{array}$ & $\begin{array}{l}99.23 \\
( \pm 13)\end{array}$ & $\begin{array}{l}99.71 \\
( \pm 13)\end{array}$ & $\begin{array}{l}94.66 \\
( \pm 13)\end{array}$ & $\begin{array}{l}100.09 \\
( \pm 13)\end{array}$ & $\begin{array}{c}110.61 \\
( \pm 13)\end{array}$ & $\begin{array}{l}110.80 \\
( \pm 13)\end{array}$ & $\begin{array}{l}110.98 \\
( \pm 13)\end{array}$ & $\begin{array}{l}90.32 \\
( \pm 13)\end{array}$ & $\begin{array}{l}79.02 \\
( \pm 13)\end{array}$ & $\begin{array}{l}76.31 \\
( \pm 13)\end{array}$ \\
\hline $\begin{array}{l}\operatorname{LogBBB}^{c} \\
(\text { PPB \%) })^{d}\end{array}$ & $\begin{array}{c}0.32 \\
(96.63)\end{array}$ & $\begin{array}{c}-0.32 \\
(73.54)\end{array}$ & $\begin{array}{c}0.34 \\
(87.38)\end{array}$ & $\begin{array}{c}0.43 \\
(96.07)\end{array}$ & $\begin{array}{c}-0.57 \\
(98.65)\end{array}$ & $\begin{array}{c}-0.90 \\
(98.87)\end{array}$ & $\begin{array}{c}-1.03 \\
(98.96)\end{array}$ & $\begin{array}{c}-0.16 \\
(88.70)\end{array}$ & $\begin{array}{c}-0.06 \\
(95.03)\end{array}$ & $\begin{array}{c}-0.62 \\
(88.57)\end{array}$ & $\begin{array}{c}-0.06 \\
(99.21)\end{array}$ & $\begin{array}{c}0.25 \\
(87.66)\end{array}$ & $\begin{array}{l}-0.10 \\
(91.16)\end{array}$ \\
\hline $\begin{array}{l}\mathrm{LD}_{50} \text { rat/mouse (mg } \\
\mathrm{kg}^{-1} \text {, oral) }\end{array}$ & $560 / 860$ & $500 / 690$ & $280 / 420$ & $290 / 460$ & $400 / 730$ & $390 / 720$ & $380 / 700$ & $310 / 570$ & $310 / 620$ & $290 / 530$ & $640 / 530$ & $620 / 960$ & $470 / 790$ \\
\hline $\begin{array}{l}\mathbf{L} \mathbf{D}_{50} \text { ratmouse (mg } \\
\mathbf{k g}^{-1} \text {, } \\
\text { intraperitioneal) }\end{array}$ & $1500 / 2200$ & $1500 / 1700$ & $770 / 1100$ & $710 / 1100$ & $1000 / 2000$ & $980 / 1400$ & $940 / 2100$ & $1100 / 1700$ & $980 / 1800$ & $1000 / 1900$ & $1100 / 1600$ & $1900 / 1900$ & $1900 / 2300$ \\
\hline $\begin{array}{l}\mathbf{L D}_{50 \text { mouse }}\left(\mathrm{mg} \mathrm{kg}^{-1} \text {, }\right. \\
\text { intravenous) }\end{array}$ & 130 & 150 & 110 & 100 & 170 & 150 & 150 & 120 & 110 & 100 & 110 & 170 & 100 \\
\hline $\begin{array}{l}\mathbf{L D}_{50 \text { mouse }}\left(\mathbf{m g} \mathbf{k g}^{-1} \text {, }\right. \\
\text { subcutaneous) }\end{array}$ & 1300 & 850 & 570 & 610 & 660 & 620 & 570 & 660 & 700 & 580 & 550 & 1300 & 730 \\
\hline $\begin{array}{l}\text { Ames test } \\
\text { (genotoxicity, \%) }\end{array}$ & 0.007 & 0.008 & 0.015 & 0.011 & 0.001 & 0.002 & 0.001 & 0.012 & 0.009 & 0.013 & 0.002 & 0.006 & 0.399 \\
\hline $\begin{array}{l}\text { Prob. of blood } \\
\text { effect }\end{array}$ & 0.53 & 0.38 & 0.56 & 0.61 & 0.36 & 0.34 & 0.31 & 0.31 & 0.36 & 0.26 & 0.37 & 0.42 & 0.37 \\
\hline $\begin{array}{l}\text { Prob. of } \\
\text { cardiovascular } \\
\text { system }\end{array}$ & 0.84 & 0.78 & 0.75 & 0.76 & 0.27 & 0.22 & 0.28 & 0.77 & 0.80 & 0.79 & 0.48 & 0.80 & 0.94 \\
\hline $\begin{array}{l}\text { Prob. of } \\
\text { gastrointestinal } \\
\text { system }\end{array}$ & 0.88 & 0.82 & 0.93 & 0.99 & 0.74 & 0.74 & 0.71 & 0.70 & 0.73 & 0.67 & 0.84 & 0.47 & 0.89 \\
\hline $\begin{array}{l}\text { Prob. of kidney } \\
\text { effect }\end{array}$ & 0.25 & 0.15 & 0.20 & 0.22 & 0.10 & 0.14 & 0.13 & 0.12 & 0.14 & 0.10 & 0.24 & 0.21 & 0.20 \\
\hline $\begin{array}{l}\text { Prob. of liver } \\
\text { effect }\end{array}$ & 0.34 & 0.60 & 0.53 & 0.57 & 0.12 & 0.13 & 0.36 & 0.12 & 0.13 & 0.35 & 0.18 & 0.25 & 0.65 \\
\hline $\begin{array}{l}\text { Prob. of lung } \\
\text { effect }\end{array}$ & 0.31 & 0.26 & 0.58 & 0.57 & 0.20 & 0.20 & 0.18 & 0.18 & 0.19 & 0.22 & 0.50 & 0.29 & 0.17 \\
\hline
\end{tabular}

${ }^{\mathrm{a}}$ Human oral bioavailability, ${ }^{\mathrm{b}} \mathrm{Human}$ intestinal absorption., ${ }^{\mathrm{C}}$ Blood Brain Barrier, ${ }^{\mathrm{d}}$ Plasma protein binding. 


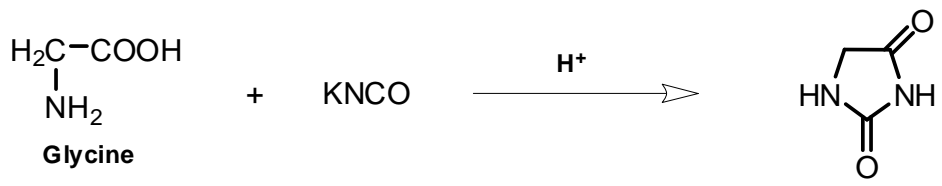

1
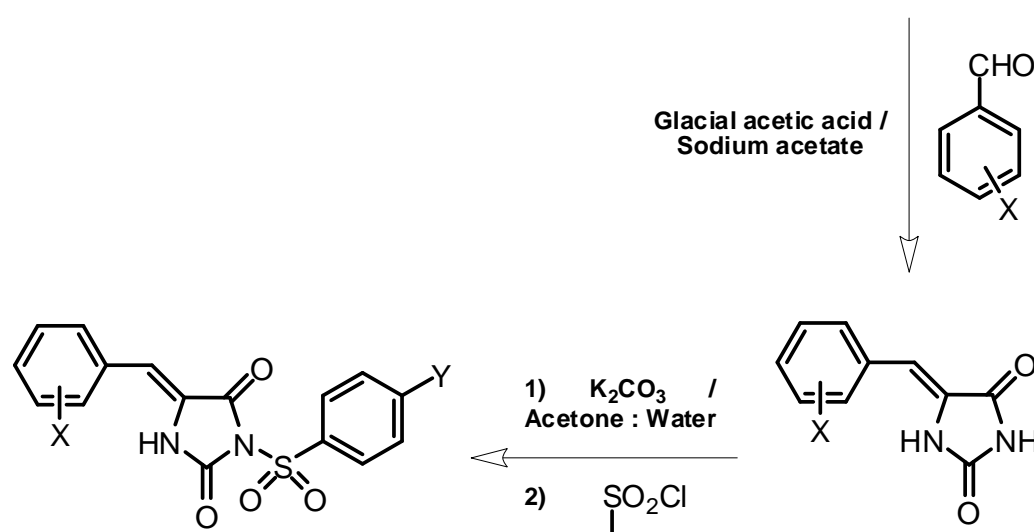

3a-r

1) $\mathrm{K}_{2} \mathrm{CO}_{3} \quad$ I Acetone : Water

2)

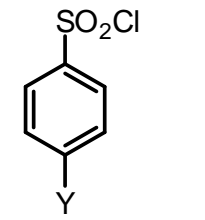

2a-f

$\mathrm{X}=\mathrm{H}, 4-\mathrm{Br}, 4-\mathrm{Cl}, 3-\mathrm{NO}_{2}, 4-\mathrm{OCH}_{3}, 4-\mathrm{N}\left(\mathrm{CH}_{3}\right)_{2}$ $\mathrm{Y}=\mathrm{H}, \mathrm{Cl}, \mathrm{CH}_{3}$

Scheme 1. Synthesis of 5-(substituted)benzylidene-3-(4-substituted)phenylsulfonyl imidazolidine-2,4-diones 3a-r. 


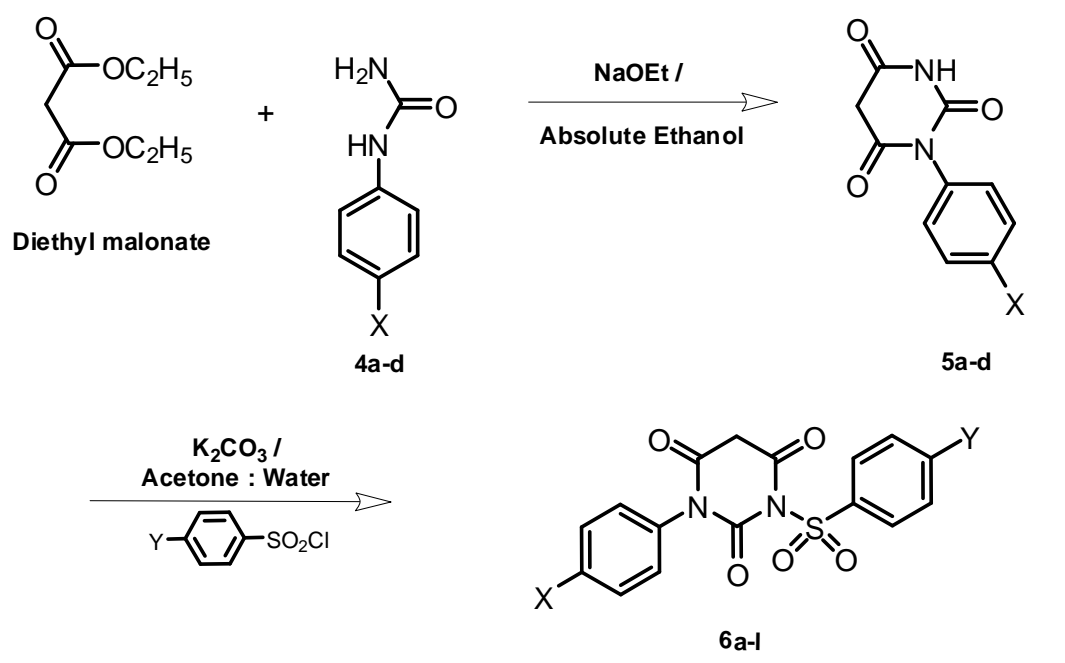

$$
\begin{aligned}
& \mathrm{X}=\mathrm{H}, \mathrm{Cl}, \mathrm{CH}_{3}, \mathrm{OCH}_{3} \\
& \mathrm{Y}=\mathrm{H}, \mathrm{Cl}, \mathrm{CH}_{3}
\end{aligned}
$$

Scheme 2. Synthesis of 1-(4-substituted)phenylsulfonyl-3-(4-substituted)phenylpyrimidine-2,4,6-(1H,3H,5H)-triones 6a-1. 

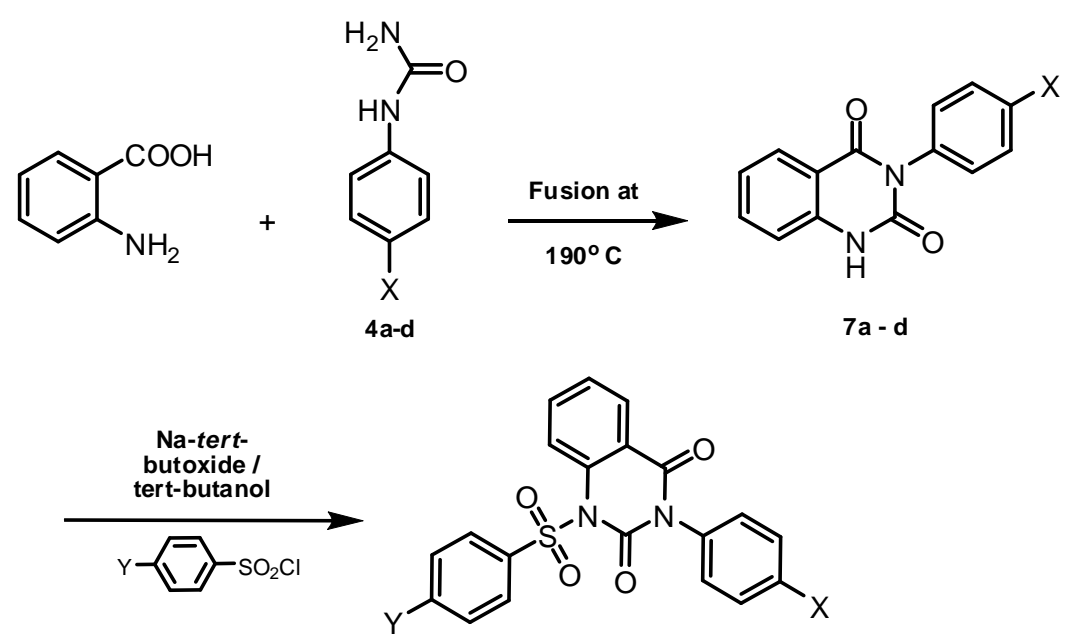

8a-1

$\mathrm{X}=\mathrm{H}, \mathrm{Cl}, \mathrm{CH}_{3}, \mathrm{OCH}_{3}$

$\mathrm{Y}=\mathrm{H}, \mathrm{Cl}, \mathrm{CH}_{3}$

Scheme 3. Synthesis of 3-(4-substituted)phenyl-1-(4-substituted)phenylsulfonylquinazoline-2,4(1H,3H)-diones 8a-l. 
<smiles>O=C(Nc1ccc(Cl)cc1)NS(=O)(=O)c1ccc2c(c1)CCC2</smiles>

LT186641 (A, Sulof enur)<smiles>O=S(=O)(NC(=S)Nc1ccc(Cl)cc1)c1ccc2c(c1)CCC2</smiles>

(B)<smiles>O=C(Nc1ccc(Cl)c(Cl)c1)NS(=O)(=O)c1ccc2c(c1)CCO2</smiles>

LY295501 (C)<smiles>Nc1ccc(C(=O)N2CCc3cc(S(=O)(=O)N4CC(c5ccccc5)NC4=O)ccc32)cc1</smiles>

4-Phenylimidazolidine-2-one (D)<smiles></smiles>

3<smiles>[X]c1ccc(N2C(=O)CC(=O)N(S(=O)(=O)c3ccc([X])cc3)C2=O)cc1</smiles>

6<smiles>[X]c1ccc(-n2c(=O)c3ccccc3n(S(=O)(=O)c3ccc([X])cc3)c2=O)cc1</smiles>

8

Figure 1. Reported (A - E) and proposed (3, 6 and 8) diarylsulfonylureas. 


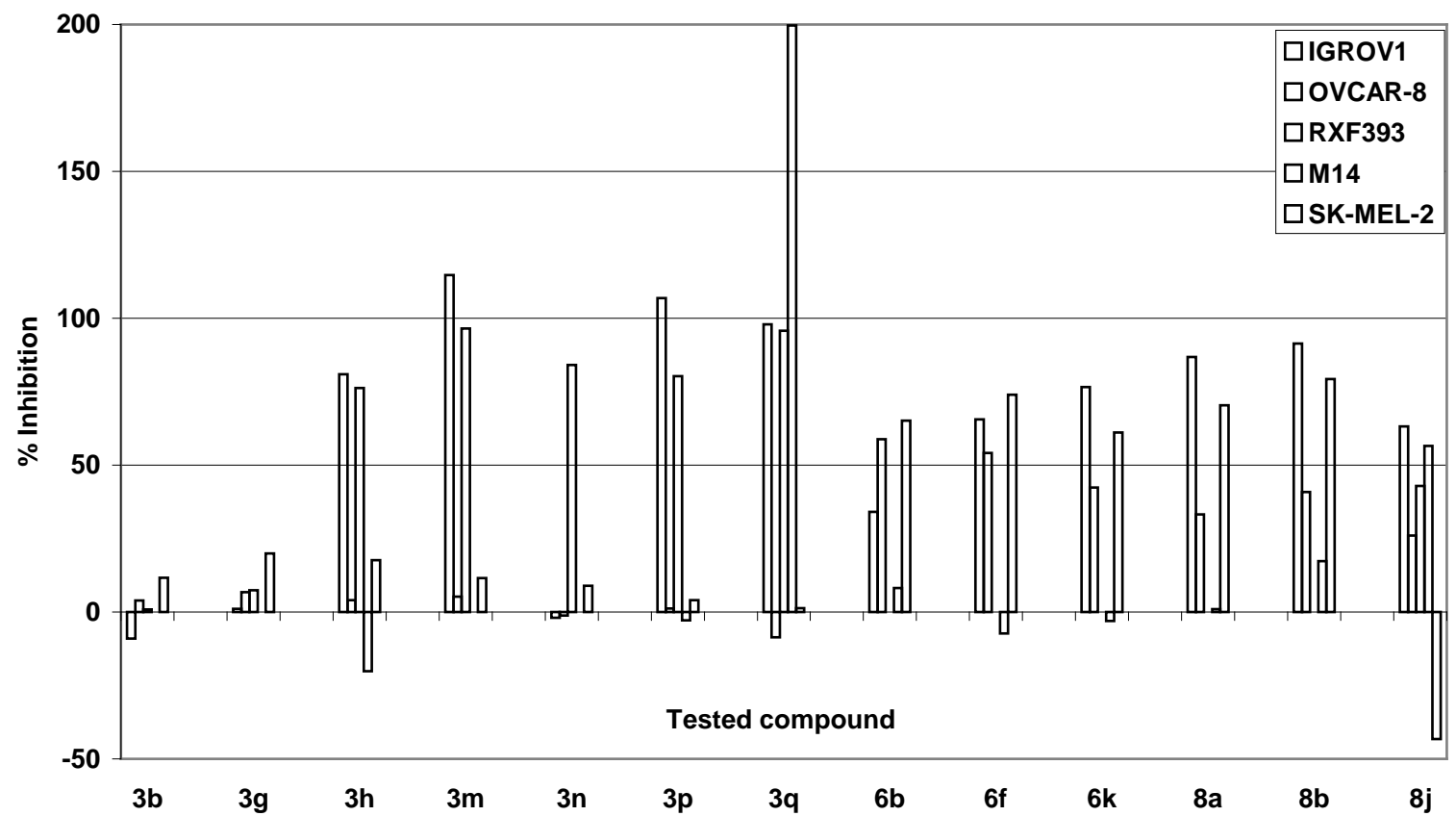

Figure 2. The percentages of growth inhibition of the 13 selected compounds over the most sensitive tumor cell lines. 


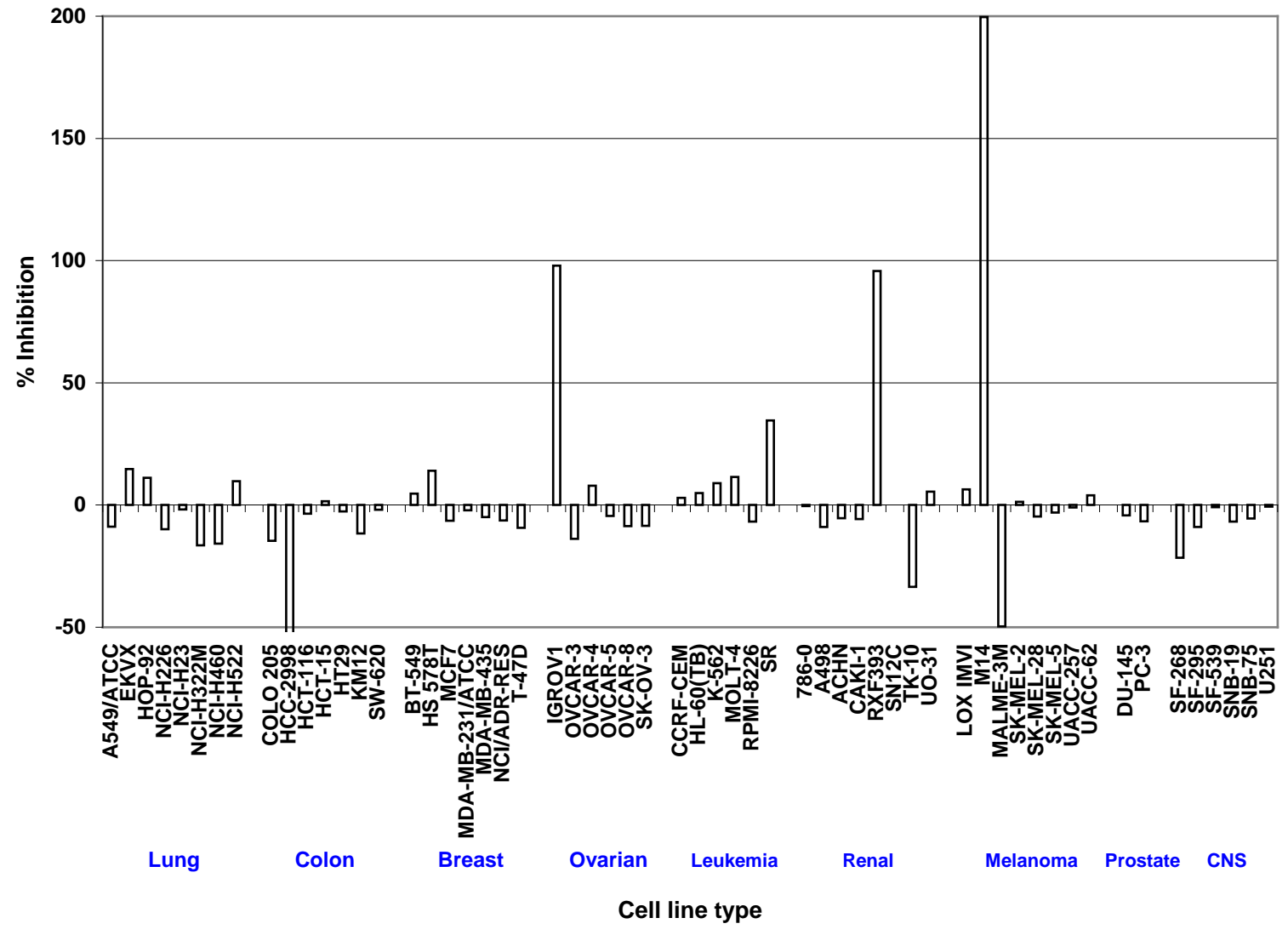

Figure 3. The percentages of growth inhibition of compound 3q over the full panel of tumor cell lines. 

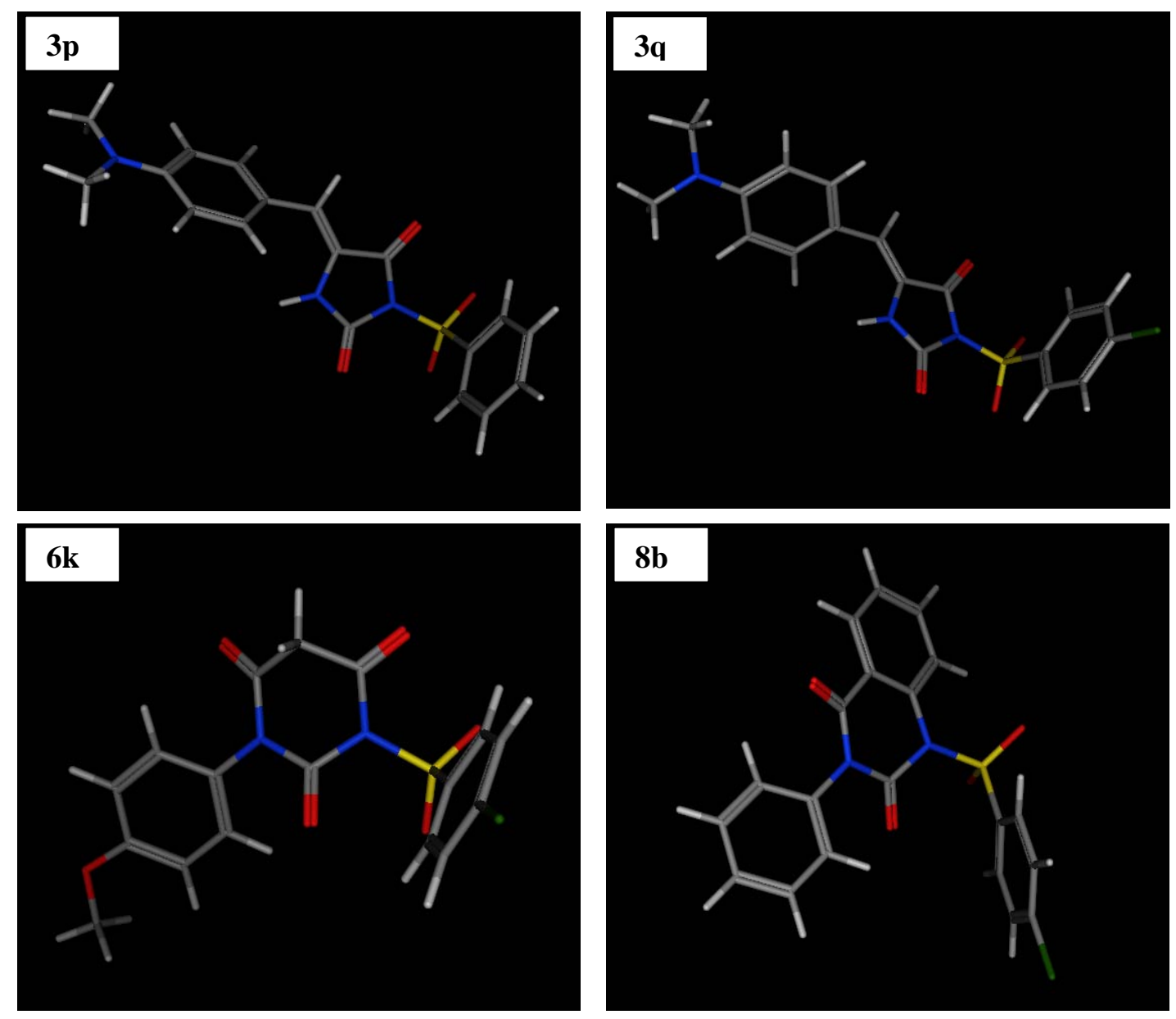

Figure 4. Lowest energy conformers of the most active compounds $\mathbf{3 p}, \mathbf{3 q}, \mathbf{6 k}$ and $\mathbf{8 b}$ as representative examples. 


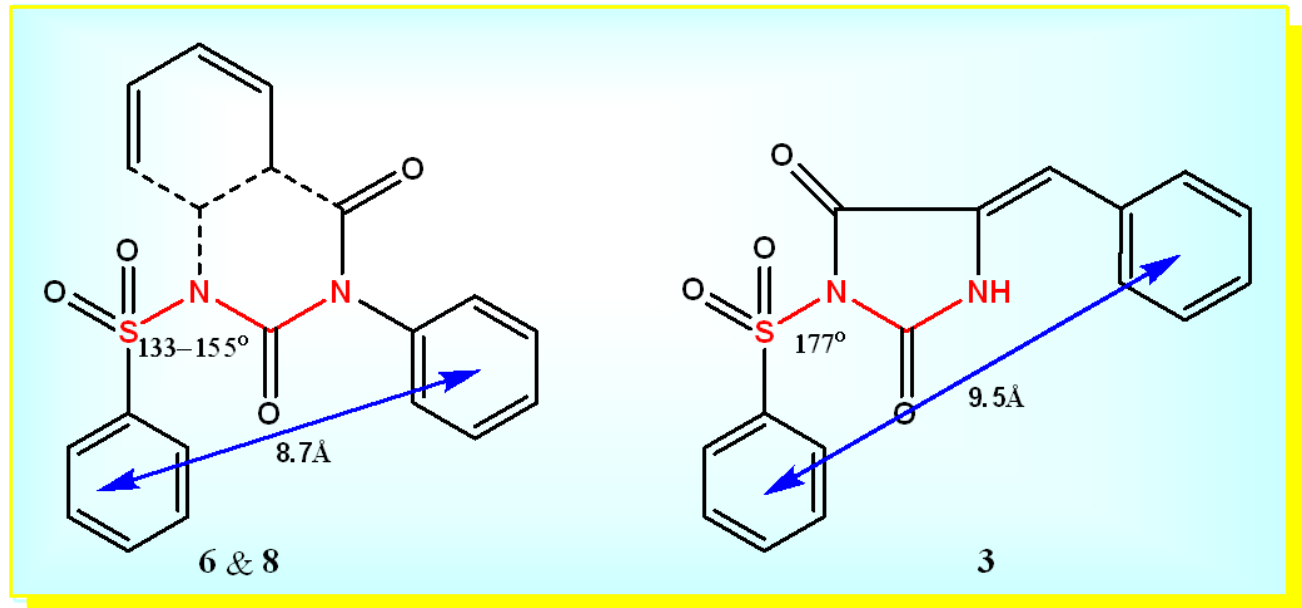

Figure 5. Deduced 2D-cyclic arylsulfonylurea pharmacophore. 

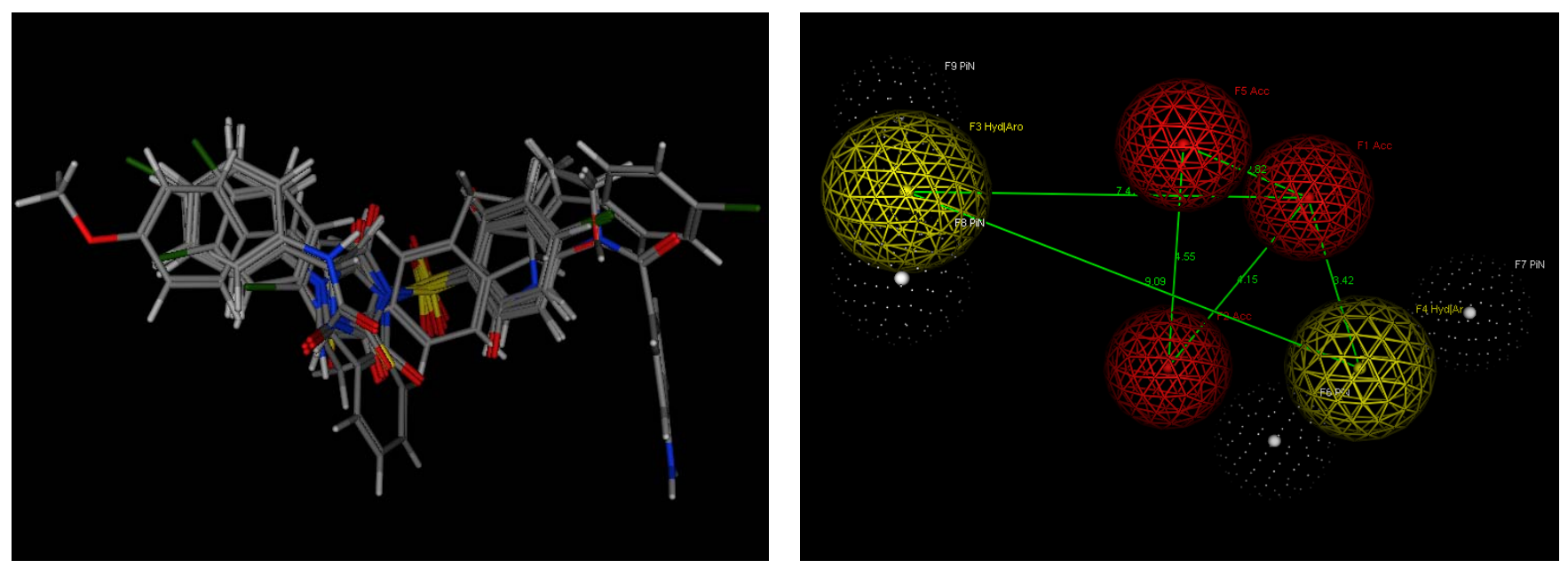

Figure 6. Alignment of a training set (Left panel) and the hypothetical 3D-pharmacophore geometry developed for the proposed cyclic arylsulfonylurea. (Right panel).

F1: Hydrogen bond acceptor center; F2: Aromatic or hydrophobic center; F3 and F5: Aromatic or Pi orbital place at the receptor site; F4: Aromatic center carrying a H-bond donor; F6: Hydrophobic center; F7: H-bond acceptor place at the receptor site. 

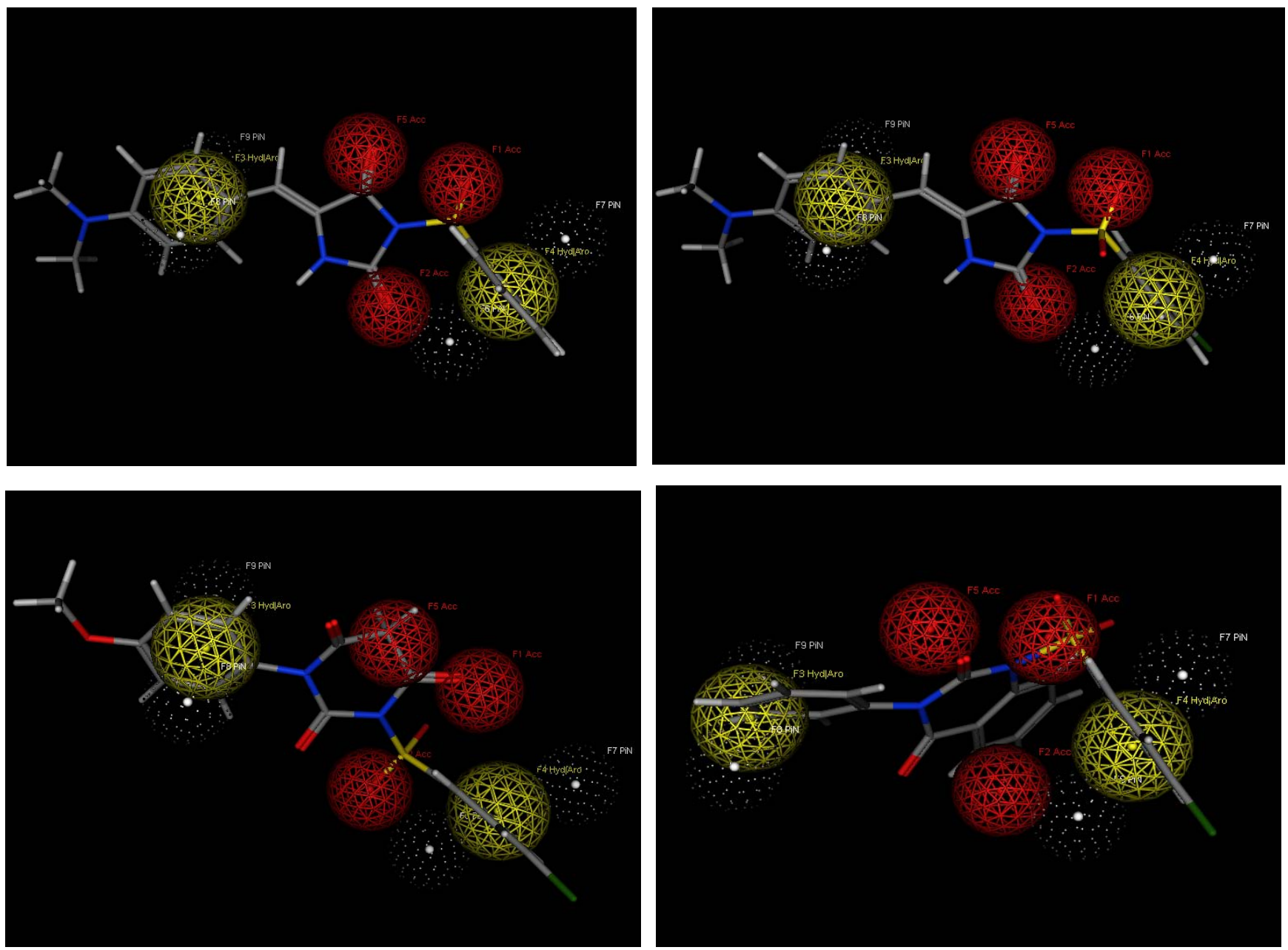

Figure 7. Superimposition of the energy-minimized structures of compounds 3p (Upper left panel), 3q (Upper right panel), 6k (Lower left panel) and $\mathbf{8 b}$ (Lower right panel) on the hypothetical 3D-pharmacophore geometry developed for the proposed cyclic arylsulfonylurea. 